\title{
FIBRATIONS OF ORDERED GROUPOIDS AND THE FACTORIZATION OF ORDERED FUNCTORS
}

\author{
NOUF ALYAMANI, N. D. GILBERT, AND E.C. MILLER.
}

\begin{abstract}
We investigate canonical factorizations of ordered functors of ordered groupoids through star-surjective functors. Our main construction is a quotient ordered groupoid, depending on an ordered version of the notion of normal subgroupoid, that results is the factorization of an ordered functor as a star-surjective functor followed by a star-injective functor. Any star-injective functor possesses a universal factorization through a covering, by Ehresmann's Maximum Enlargement Theorem. We also show that any ordered functor has a canonical factorization through a functor with the ordered homotopy lifting property.
\end{abstract}

\section{INTRODUCTION}

The idea of treating a groupoid - a small category in which every arrow is invertible - as an algebraic structure has been established as a profitable approach to problems in group theory, where groupoids offer algebraic analogues of relevant notions from the homotopy theory of paths and covering spaces, and applications of group theory can often be more conveniently phrased in terms of groupoids. The book [6] by P.J.Higgins is an admirable introduction. The role of groupoids in describing symmetry is concisely surveyed in [13].

In the 1950's, C. Ehresmann developed a new foundation of differential geometry based on groupoids [4]. His algebraic model of a pseudogroup of transformations was an ordered groupoid, in which the key structural idea corresponds to the restriction of domain. The introduction of an ordered structure leads to a separation from group theory since a group, when considered as an ordered groupoid, can only carry the equality ordering. Instead, the most closely related algebraic structure is that of an inverse semigroup. Inverse semigroups correspond to the subclass of inductive groupoids, in which the poset of identity elements forms a semilattice. The consequent close interactions between the theories of ordered groupoids and inverse semigroups are a major theme of the book [7] of Lawson.

2010 Mathematics Subject Classification. Primary: 20L05; Secondary 18D15,55U35.

Key words and phrases. ordered groupoid, functor, fibration, covering.

The first author acknowledges the support of a PhD scholarship from the Royal Government of Saudi Arabia, held whilst this work was carried out. The third author acknowledges the financial support of a studentship from the Carnegie Trust for the Universities of Scotland. 
In this paper, we consider some of the homotopy notions modelled by groupoids, but in the context of ordered groupoids. Our starting point is the paper [1] on fibrations of groupoids by Brown, in which fundamental constructions for fibrations of groupoids are explored, and applications given to the non-abelian cohomology of groups and to track groupoids in homotopy theory. The groupoid notions of the homotopy-lifting property and the path-lifting property for a map of groupoids $p: G \rightarrow H$ are shown to be equivalent, and equivalent to $p$ being star-surjective. Any one of these properties thus defines $p$ as a fibration. Steinberg [12] adopts star-surjectivity as the definition of a fibration of ordered groupoids. He undertakes a general study of factorization theorems for ordered groupoids, based on the semidirect product construction for one ordered groupoid acting on another (also found in [2]) and a left adjoint Der - the derived ordered groupoid - to the semidirect product. Steinberg uses the derived ordered groupoid to show that every ordered groupoid morphism factors in a canonical way through a fibration, and that a star-injective morphism has a universal factorization through a covering. The latter result is Ehresmann's Maximum Enlargement Theorem [4]. Lawson's approach to this result [7] also demonstrates its applications in inverse semigroup theory, and in particular to the theory of idempotent pure homomorphisms. Another important precursor to our work is the thesis of Matthews [9] and the paper [8]. Matthews studies inverse semigroups and ordered groupoids from the point of view of abstract homotopy theory, showing that the category of ordered groupoids admits cocylinders, and as an application is able to deduce Steinberg's Fibration Theorem.

This paper focusses on fibrations of ordered groupoids, and our first significant result is that in the ordered case, the homotopy lifting property and star-surjectivity are no longer equivalent properties. Star-surjectivity is still equivalent to pathlifting, but homotopy lifting is a stronger property: we therefore consider starsurjective ordered functors - fibrations as in [1, 12] - and strong fibrations that have the homotopy lifting property. We establish some formal properties of these classes of fibration in section 2, and show in section 3 how a quotient construction for ordered groupoids leads to a factorization of any morphism as a fibration followed by a star-injective functor. This is an extension to the ordered case of a basic result for groupoid morphisms [1, Proposition 2.4], but in the ordered case requires a remodelled definition of a normal ordered subgroupoid $A$ of an ordered groupoid $G$ (more general than that of [9]) and the construction of a quotient ordered groupoid $G / / A$, for which the canonical map $G \rightarrow G / / A$ is always a fibration.

We then consider the Maximum Enlargement Theorem in section 4 and the Fibration Theorem in section 5. We give a direct proof of the Maximum Enlargement Theorem, by constructing from a star-injective morphism $U \rightarrow H$ a poset $P$ with a natural $H$-action such that the semidirect product $H \ltimes P$ is an enlargement of $U$. Coupled with our quotient construction, the Maximum Enlargement Theorem then gives a universal factorization of any ordered groupoid morphism as a fibration followed by an enlargement followed by a covering. We follow [8] and use the mapping cocylinder $M^{\phi}$ of an ordered groupoid morphism $\phi: G \rightarrow H$ in 
our discussion of the Fibration Theorem, but we show directly that the morphism $M^{\phi} \rightarrow H$ is a strong fibration. The factorization of an ordered groupoid morphism $\phi: G \rightarrow H$ as $G \rightarrow M^{\phi} \rightarrow H$ then implies that $\phi$ factorizes as an enlargement followed by a fibration followed by a covering.

\section{GROUPOIDS AND ORDERED GROUPOIDS}

A groupoid $G$ is a small category in which every morphism is invertible. We consider a groupoid as an algebraic structure (as in [6, 7]): the elements are the morphisms, and composition is an associative partial binary operation. The set of identities in $G$ is denoted $G_{0}$, and an element $g \in G$ has domain $g \mathbf{d}=g g^{-1}$ and range $g \mathbf{r}=g^{-1} g$. If every arrow in $G$ is an identity, we say that $G$ is trivial. The groupoid $\mathscr{I}$ has two identities, 0 and 1 , and two non-identity morphisms, $\iota$ and $\iota^{-1}$, with $\iota \mathbf{d}=0$ and $\iota \mathbf{r}=1$.

Let $e \in G_{0}$. Then the star of $e$ in $G$ is the set $\operatorname{star}_{G}(e)=\{g \in G: g \mathbf{d}=e\}$, and the local group at $e$ is the set $G(e)=\{g \in G: g \mathbf{d}=e=g \mathbf{r}\}$. A functor $\phi: G \rightarrow H$ is said to be star-surjective if, for each $e \in G_{0}$, the restriction $\phi: \operatorname{star}_{G}(e) \rightarrow \operatorname{star}_{H}(e \phi)$ is surjective. Star-injective and star-bijective functors are defined similarly. A star-surjective functor is also called a fibration, a starinjective functor an immersion, and a star-bijective functor a covering.

The kernel of a functor $\phi$ is the inverse image of the identities:

$$
\operatorname{ker} \phi=\left\{g \in G: g \phi \in H_{0}\right\} \text {. }
$$

Lemma 1.1. A functor $\phi: G \rightarrow H$ is star-injective if and only if its kernel is equal to $G_{0}$.

A normal subgroupoid $N$ of a groupoid $G$ is a subgroupoid such that

- $N$ is wide in $G$ : that is, $N_{0}=G_{0}$,

- if $g \in G$ and $n \in N$ with $g^{-1} n g$ defined in $G$, then $g^{-1} n g \in N$.

We remark that $g^{-1} n g$ is defined if and only if $n \in G(g \mathbf{d})$. Now $N$ may well contain elements not in local groups, and this definition follows that given by Higgins in [6]. However, we note that Matthews [9] assumes that if $n \in G$ then $n \in G(e)$ for some $e \in G_{0}$. This distinction will be of importance in section 3 , A normal subgroupoid $N$ then determines an equivalence relation $\simeq_{N}$ on $G$, defined by

$$
g \simeq_{N} h \Longleftrightarrow \text { there exist } m, n \in N \text { such that } g=m h n .
$$

The set $G / N$ of $\simeq_{N}$-equivalence classes inherits a natural groupoid structure, see [6]. The kernel of a functor $\phi: G \rightarrow H$ is a normal subgroupoid, and the canonical map $G \rightarrow G / \operatorname{ker} \phi$ is a fibration, and $\phi$ then induces an immersion $G / \operatorname{ker} \phi \rightarrow H$.

An ordered groupoid $(G, \leqslant)$ is a groupoid $G$ with a partial order $\leqslant$ satisfying the following axioms:

OG1 for all $g, h \in G$, if $g \leqslant h$ then $g^{-1} \leqslant h^{-1}$, 
OG2 if $g_{1} \leqslant g_{2}, h_{1} \leqslant h_{2}$ and if the compositions $g_{1} h_{1}$ and $g_{2} h_{2}$ are defined, then $g_{1} h_{1} \leqslant g_{2} h_{2}$,

OG3 if $g \in G$ and $f$ is an identity of $G$ with $f \leqslant g \mathbf{d}$, there exists a unique element $(f \mid g)$, called the restriction of $g$ to $f$, such that $(f \mid g) \mathbf{d}=f$ and $(f \mid g) \leqslant g$.

As a consequence of [OG3] we also have:

OG3* if $g \in G$ and $f$ is an identity of $G$ with $f \leqslant g r$, there exists a unique element $(g \mid f)$, called the corestriction of $g$ to $f$, such that $(g \mid f) \mathbf{r}=f$ and $(g \mid f) \leqslant g$,

since the corestriction of $g$ to $f$ may be defined as $\left(f \mid g^{-1}\right)^{-1}$.

Let $G$ be an ordered groupoid and let $a, b \in G$. Suppose that $a \mathbf{r}$ and $b \mathbf{d}$ have a greatest lower bound - which we write as $(a \mathbf{r})(b \mathbf{d})-$ in $G_{0}$. Then we may define the pseudoproduct of $a$ and $b$ in $G$ as

$$
a * b=(a \mid(a \mathbf{r})(b \mathbf{d}))((a \mathbf{r})(b \mathbf{d}) \mid b),
$$

where the right-hand side is a composition in the groupoid $G$. As Lawson shows in Lemma 4.1.6 of [7], this is a partially defined associative operation on $G$.

Our discussion of ordered groupoids follows the terminology and notation of [7], except that we have interchanged the use of domain and range and that we use $*$ to denote the pseudoproduct, rather than $\otimes$.

An ordered groupoid $G$ is inductive if $G_{0}$ is a meet semilattice. In this case the pseudoproduct is everywhere defined on $G$, and makes $G$ into an inverse semigroup. On the other hand, each inverse semigroup $S$ has an underlying inductive groupoid $G(S)$, whose arrows are the elements of $S$, with $s \mathbf{d}=s s^{-1}$ and $s \mathbf{r}=s^{-1} s$, and where composition is just the restriction of the multiplication in $S$. These constructions yield an isomorphism between the categories of inductive groupoids and of inverse semigroups, see [7, Theorem 4.1.8].

Example 1.2. Let $P$ be a poset and $G=(\mathscr{G}, P)$ be a presheaf of groups over $P$, with linking maps $\alpha_{y}^{x}$ for $x \geqslant y$ in $P$. Here $\mathscr{G}$ assigns a group $G_{x}$ to each $x \in P$ and $\alpha_{y}^{x}$ is a group homomorphism $G_{x} \rightarrow G_{y}$. We have $\alpha_{x}^{x}=$ id for all $x \in P$, and whenever $x \geqslant y \geqslant z$ in $P$ then $\alpha_{y}^{x} \alpha_{z}^{y}=\alpha_{z}^{x}$. The groupoid composition on $G$ is just the collection of group operations in the groups $G_{x}$, and $G$ is ordered by $g \geqslant g \alpha_{y}^{x}$ whenever $x, y \in P$ with $x \geqslant y$ and $g \in G_{x}$.

A morphism of ordered groupoids is an order-preserving functor $A \rightarrow B$. We shall call such functors simply ordered functors. We then have the category OGpd of ordered groupoids and ordered functors. The set of ordered functors $A \rightarrow B$ is denoted by $\operatorname{OGpd}(A, B)$. We note that the category of ordered groupoids is cartesian closed. So given ordered groupoids $A, B$ there exists an ordered groupoid $\operatorname{OGPD}(A, B)$ such that there is a natural equivalence

$$
\operatorname{OGPD}(A \times B, C) \rightarrow \operatorname{OGPD}(B, \operatorname{OGPD}(A, C)) .
$$


The object set of $\operatorname{OGPD}(A, B)$ is $\operatorname{OGpd}(A, B)$. Given two ordered functors $f, g$ : $A \rightarrow B$, an arrow in $\operatorname{OGPD}(A, B)$ from $f$ to $g$ is an ordered natural transformation $\tau$ from $f$ to $g$ : that is, $\tau$ is an ordered function $\operatorname{obj}(A) \rightarrow B$ such that, for each arrow $a \in A$ with $a \mathbf{d}=x$ and $a \mathbf{r}=y$, the square

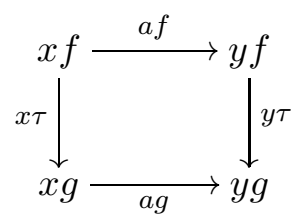

in $B$ commutes.

\section{FiBRATIONS}

Let $p: G \rightarrow H$ be a morphism of groupoids. Then $p$ has the homotopy lifting property if, given any groupoid $A$ and a commutative square

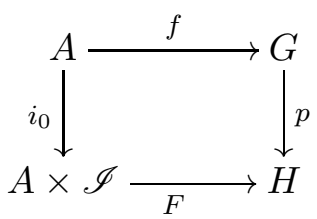

where $a i_{0}=(a, 0)$, there exists a morphism $\widetilde{F}: A \times \mathscr{I} \rightarrow G$ such that the diagram

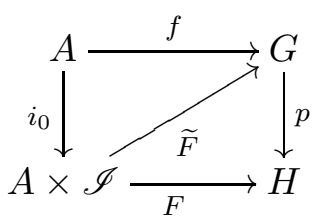

commutes. Proposition 2.1 of Brown's paper [1] shows that possession of this property by $p$ is equivalent to $p$ being star-surjective, and so drawing on the evident topological analogy, Brown calls star-surjective morphisms fibrations of groupoids. In fact, [1, Proposition 2.1] shows the equivalence of the homotopy lifting property, the path-lifting property, and star-surjectivity. The path-lifting property is the special case of the homotopy lifting property in which the groupoid $A$ is a trivial groupoid with one identity.

The homotopy lifting property and the path-lifting property for ordered groupoids are formulated by repeating the above definitions with the addition that every morphism should be a morphism of ordered groupoids. A distinction then arises between the homotopy lifting property and being star-surjective.

Proposition 2.1. [8, Proposition 4.7] A morphism of ordered groupoids has the path lifting property if and only if it is star-surjective: in particular, a morphism of ordered groupoids with the homotopy lifting property is star-surjective. 
Proof. As already remarked, the path-lifting property is a special case of the homotopy lifting property. So suppose that $p: G \rightarrow H$ has the path lifting property. Let $e$ be an identity of $G$ and suppose that $h \in \operatorname{star}_{H}(e p)$. Let 0 denote a trivial groupoid with a single identity. Any morphism from $0 \times \mathscr{I} \rightarrow H$ is determined by the choice of a single arrow in $H$, and will be ordered. Thus $e$ determines a morphism $0 \rightarrow G$ and $h$ determines a morphism $0 \times \mathscr{I} \rightarrow H$ making the commutative square

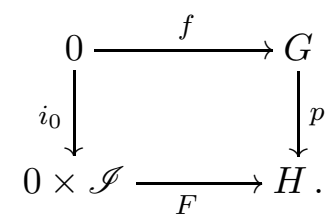

The lifting $\widetilde{F}: 0 \times A \rightarrow G$ in the commutative diagram

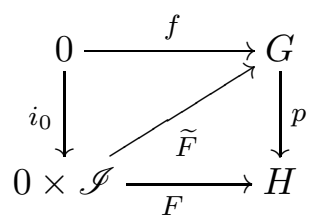

is then determined by an arrow $g \in G$ satisfying $g \mathbf{d}=e$ and $g p=h$. Therefore $p$ is star-surjective.

For the converse, given a commutative square

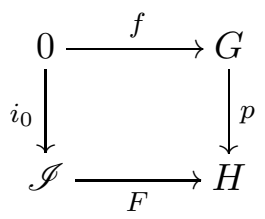

with $p$ star-surjective, we obtain a morphism $\widetilde{F}: \mathscr{I} \rightarrow G$ making

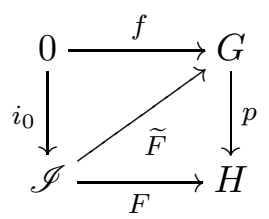

commute by defining $\iota \widetilde{F}$ to be any arrow in $\operatorname{star}_{G}(0 f)$ mapping to $\iota F \in H$. Since $\mathscr{I}$ carries the trivial ordering, any choice for $\iota \widetilde{F}$ determines an ordered morphism.

However, a star-surjective ordered morphism need not have the ordered homotopy lifting property, as the following counterexample shows.

Example 2.2. Let $E=\{e, f, z\}$ be the semilattice with $e \geqslant z \leqslant f$. We take $G$ to be a semilattice of groups over $E$ with linking maps $\alpha_{z}^{e}: G_{e} \rightarrow G_{z}$ and $\alpha_{z}^{f}: G_{f} \rightarrow G_{z}$, and we take $H$ to be a semilattice of groups over the semilattice 
$\{0,1\}$, where $0<1$, with linking map $\psi=\psi_{0}^{1}: H_{1} \rightarrow H_{0}$. Let $i: E \rightarrow G$ be the inclusion map, and let $p: G \rightarrow H$ be the star-surjective morphism determined by three surjections $p_{e}: G_{e} \rightarrow H_{1}, p_{f}: G_{f} \rightarrow H_{1}$ and $p_{z}: G_{z} \rightarrow H_{0}$. Hence the following diagram commutes:

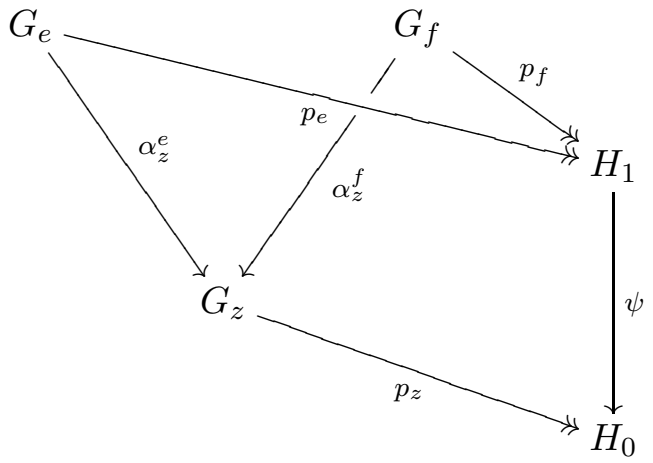

A homotopy $F: E \times \mathscr{I} \rightarrow H$ is determined by two elements $h_{e}, h_{f} \in H$ satisfying $h_{e} \psi=h_{f} \psi$, and then $(e, \iota) F=h_{e},(f, \iota) F=h_{f}$ and $(z, \iota) F=h_{e} \psi=h_{f} \psi$.

To construct the lifting homotopy $\widetilde{F}: E \times \mathscr{I} \rightarrow G$ we are required to find $g_{e}, g_{f} \in$ $G$ such that

$$
\begin{aligned}
& \text { - } g_{e} \alpha_{z}^{e}=g_{f} \alpha_{z}^{f} \\
& \text { - } g_{e} p_{e}=h_{e} \text { and } g_{f} p_{f}=h_{f} \\
& \text { - } g_{e} \alpha_{z}^{e} p_{z}=g_{f} \alpha_{z}^{f} p_{z}=h_{e} \psi=h_{f} \psi \text {. }
\end{aligned}
$$

Only the first of these conditions is imposed by the requirement that $\widetilde{F}$ be ordered. To fulfill the second and third conditions we can choose any preimages $g_{e}, g_{f}$ of $h_{e}, h_{f}$ under $p_{e}, p_{f}$ to satisfy the second condition, and commutativity of (2.1) guarantees the third condition.

However, we cannot always make a consistent choice of $g_{e}, g_{f}$ to satisfy all three conditions. Take $G_{e}=\{1, a\}, G_{f}=\{1, b\}$ be cyclic groups of order two and let $G_{z}$ be the Klein four-group $\{1, a, b, a b\}$ with $\alpha_{z}^{e}, \alpha_{z}^{f}$ the inclusions. Take $H_{1}=$ $\{1, x\}$ and $H_{0}=\{1, y\}$ also cyclic of order two, with $\psi: x \mapsto y$. Let $p_{e}: a \mapsto$ $x, p_{f}: b \mapsto x$ and $p_{z}: a \mapsto y, b \mapsto y$. If we now choose $h_{e}=x=h_{f}$ then we have to take $g_{e}=a$ and $g_{f}=b$ as preimages: but then $g_{e} \alpha_{z}^{e}=a \neq b=g_{f} \alpha_{z}^{f}$. Hence no ordered lifting homotopy exists.

The distinction between ordered fibrations and ordered morphisms with the ordered homotopy lifting property was raised but left undecided in [9]. We shall follow Steinberg [12] is defining a fibration of ordered groupoids to be a star-surjective ordered morphism. A strong fibration is defined to be a morphism of ordered groupoids that has the homotopy lifting property. We note a couple of constructions for fibrations.

Proposition 2.3. $\quad$ (i) An ordered covering $p: G \rightarrow H$ is a strong fibration, 
(ii) A pullback of a fibration is a fibration,

(iii) A pullback of a strong fibration is a strong fibration.

Proof. (i) We wish to construct $\widetilde{F}$ in a diagram

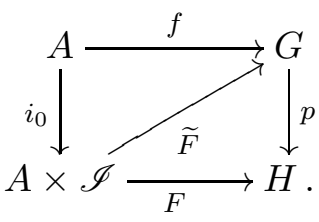

For $a \in A$ we must have $(a, 0) \widetilde{F}=a f$. Now for any $x \in A_{0}$ we have $(x, 0) F=$ $x i_{0} F=x f p$ and since $p$ is a covering, there is a unique $g \in \operatorname{star}_{G}(x f)$ with $g p=(x, \iota) F$. We set $(x, \iota) \widetilde{F}=g$. Suppose that $y \leqslant x$ in $A_{0}$. Then $y f \leqslant x f$ and $(y f \mid g) p \leqslant g p=(x, \iota) F$ with $(y f \mid g) \mathbf{d}=y f$. But also $(y, \iota) F \leqslant(x, \iota) F$ with $(y, \iota) F \mathbf{d}=y f$. By uniqueness of restriction we have $(y, \iota) \widetilde{F}=(y f \mid g) \leqslant g$. We have now defined $\widetilde{F}$ consistently on $A \times\{0\}$ and on $A_{0} \times \mathscr{I}$ : these definitions combine to give $(a, \iota) \widetilde{F}=(a f) g$ where $g$ is the unique arrow in $\operatorname{star}_{G}((a \mathbf{r}) f)$ with $g p=(a \mathbf{r}, \iota) F$. This defines an ordered lifting $\widetilde{F}$ of $F$.

(ii) and (iii) The pullback results are straightforward.

Proposition 2.4. Suppose that a strong fibration $p: G \rightarrow H$ factors as the composite of a morphism $\pi: G \rightarrow T$ and an immersion $\psi: T \rightarrow H$. Then $\pi$ is a strong fibration.

Proof. Consider a commutative square

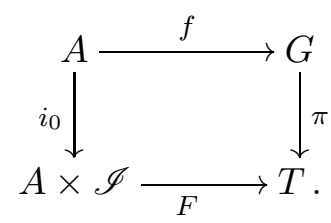

Extend this diagram by $\psi$ and use the homotopy lifting property of $p=\pi \psi$ to lift $F_{*}=F \psi$ to $G$ :

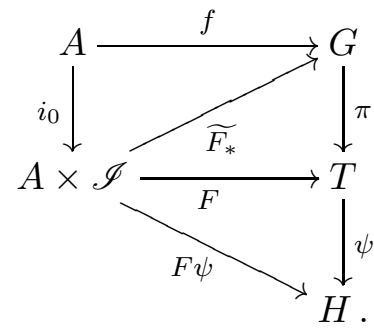

Then $i_{0} \widetilde{F}_{*}=f$. Consider $(a, \iota) \in A \times \mathscr{I}$. Then

$$
\left((a, \iota) \widetilde{F_{*}} \pi\right) \mathbf{d}=(a \mathbf{d}, 0) \widetilde{F_{*}} \pi=a \mathbf{d} f \pi=(a \mathbf{d}, 0) F=((a, \iota) \mathbf{d}) F=((a, \iota) F) \mathbf{d} .
$$


Now $(a, \iota) \widetilde{F_{*}} \pi \psi=(a, \iota) F \psi$, and since $\psi$ is an immersion, $(a, \iota) \widetilde{F_{*}} \pi=(a, \iota) F$. Hence $\widetilde{F_{*}}$ lifts $F$.

Proposition 2.5. Let $p: G \rightarrow H$ be a strong fibration of ordered groupoids. Then for any ordered groupoid $T$, the functor $p_{*}: \operatorname{OGPD}(T, G) \rightarrow \operatorname{OGPD}(T, H)$ induced by composition with $p$ is a strong fibration.

Proof. Suppose we are given a commutative square

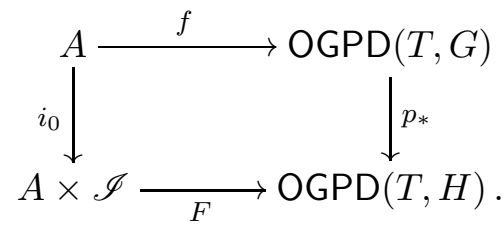

The natural equivalence (1.1) implies that there exists a corresponding commutative diagram

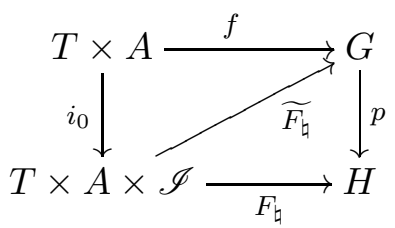

in which $F_{\natural}$ lifts to $\widetilde{F}_{\natural}$. The functor $\widetilde{F}_{\natural}$ then corresponds to $\widetilde{F}: A \times I \rightarrow$ $\operatorname{OGPD}(T, G)$ making (2.2) commute.

Example 2.6. If $p: G \rightarrow H$ is star-surjective, then $p_{*}: \operatorname{OGPD}(T, G) \rightarrow$ $\operatorname{OGPD}(T, H)$ need not be star-surjective. We take $E, G, H$ as in Example 2.2, $T=E$, and the inclusion $i: E \rightarrow G$ in $\operatorname{OGPD}(E, G)_{0}$. A natural transformation from the functor ip $\in \operatorname{OGPD}(E, H)_{0}$ is an ordered mapping $\tau: E \rightarrow H$ such that, for all $v \in E$ we have $v \tau \in H_{v p}$. We take $e \tau=x=f \tau$ and $z \tau=y$. Then a $\tilde{\tau} \in \operatorname{OGPD}(E, G)$ starting at $i$ with $\tilde{\tau} p=\tau$ must satisfy $v \tilde{\tau} \in G_{v}$ : hence $e \tilde{\tau}=a$, $f \tilde{\tau}=b$, and $z \tilde{\tau} \neq 1$. But as before we cannot choose $z \tilde{\tau} \in\{a, b, a b\}$ to obtain $z \tilde{\tau} \leqslant a$ and $z \tilde{\tau} \leqslant b$.

In the unordered setting, as noted in section 1 any groupoid morphism $\theta: G \rightarrow H$ factorises as the composition of a canonical fibration $G \rightarrow G / \operatorname{ker} \theta$ followed by a star-injective morphism $G / \operatorname{ker} \theta \rightarrow H$. However, if $\theta: G \rightarrow H$ is ordered, the quotient groupoid $G / \operatorname{ker} \theta$ may not admit an ordering so that we get an ordered fibration $G \rightarrow G / \operatorname{ker} \theta$.

Example 2.7. Consider the simplicial groupoid $B=\mathbb{N} \times \mathbb{N}$ in which $\mathbb{N}$ carries the reverse of its usual total order. We order arrows in $B$ by $(p+m, p+n) \leqslant$ $(m, n)$ for all $p, m, n \in \mathbb{N}$ : hence $(0,0)$ is a maximum element. As a poset, $\mathbb{N}$ is now an infinite descending chain, and so $B$ is inductive. The inverse semigroup corresponding to $B$ is the bicyclic monoid (see [7, section3.4]). We define $\theta$ : $B \rightarrow \mathbb{Z}_{2}$ by $(m, n) \mapsto[m-n]_{2}$, so that $\operatorname{ker} \theta=\{(m, n): m-n$ is even $\}$. Then $B / \operatorname{ker} \theta$ is isomorphic to the groupoid $\mathscr{I}$ which can only carry the trivial ordering, and hence $B \rightarrow \mathscr{I}$ is not an ordered functor. 
In the next section we shall present a modified quotient construction for ordered groupoids that leads to an ordered factorisation of an ordered functor $\theta: G \rightarrow H$.

\section{QUOTIENTS OF ORDERED GROUPOIDS}

Definition 3.1. A subgroupoid $A$ of an ordered groupoid $G$ is an normal ordered subgroupoid if

- $A$ is wide in $G$,

- if $a \in A$ and $e \leqslant a a^{-1}$ then the restriction $(e \mid a) \in A$,

- if $a \in A$ and $h, k \in G$ satisfy

$\diamond h \leqslant g$ and $k \leqslant g$ for some $g \in G$ (that is, $h$ and $k$ have an upper bound in $G$ ),

$\diamond h^{-1} a k$ is defined in $G$, then $h^{-1} a k \in A$.

In the unordered case (when $\leqslant$ is equality) we recover Higgins' definition [6] of a normal subgroupoid: the second condition is then vacuous, and in the third condition we have $h=k$ and then $h^{-1} a k$ being defined forces $a$ to be in a local subgroup of $G$. In the ordered case, if $A$ is a disjoint union of groups, then $h^{-1} a k$ being defined implies that $h h^{-1}=k k^{-1}$, and then each of $h, k$ is the restriction of $g$, so by uniqueness of restriction, $h=k$ again and we recover Matthews' definition [9].

Lemma 3.2. The kernel of an ordered morphism $\theta: G \rightarrow H$ is a normal ordered subgroupoid of $G$.

Proof. Clearly $G_{0} \subseteq \operatorname{ker} \theta$, so suppose that $a \in \operatorname{ker} \theta, h, k \in G$ with the composition $h^{-1} a k$ defined in $G$, and that there exists $g \in G$ with $h \leqslant g$ and $k \leqslant g$. Suppose that $a \theta=z \in H_{0}$. Then $\left(h^{-1} \theta\right)(k \theta)$ is defined in $H$, and $h \theta \leqslant g \theta, k \theta \leqslant g \theta$. It follows that $h \theta=(z \mid g \theta)=k \theta$ and hence

$$
\left(h^{-1} a k\right) \theta=\left(h^{-1} \theta\right)(k \theta)=(h \theta)^{-1}(k \theta) \in H_{0}
$$

and so $h^{-1} a k \in \operatorname{ker} \theta$.

Example 3.3. An inverse subsemigroup $K$ of an inverse semigroup $S$ is normal (as in [7]) if and only if $G(K)$ is a normal ordered subgroupoid of $G(S)$. For suppose that $G(K)$ is normal in $G(S)$. Consider $s^{-1} k s$ with $k \in K$ and $s \in S$. We can write, in $S$ :

$$
s^{-1} k s=\left(s^{-1} k s s^{-1} k^{-1}\right)\left(s s^{-1} k s s^{-1}\right)\left(k^{-1} s s^{-1} k s\right)
$$

and the three bracketed terms on the right-hand side are now three composable arrows in $G(S)$. The middle term is $\leqslant k$ and so is in $G(K)$. The first term is $\leqslant s^{-1}$ and the third term is $\leqslant s$ and so the whole product is in $G(K)$ and so, as an element of $S$, is in $K$. On the other hand, if $K$ is a normal inverse subsemigroup of $S$, then consider a composition $s^{-1} k t$ defined in $G(K)$ and where $s \leqslant u, t \leqslant u$ for some $u \in G$. Then in $K$ we have $s^{-1} k t \leqslant u^{-1} k u$ and $u^{-1} k u \in K$ by 
normality. So the composed arrow $u^{-1} k u \in G(K)$ and hence so is $s^{-1} k t$ since $s^{-1} k t \leqslant u^{-1} k u$.

Lemma 3.4. Let $A$ be a normal ordered subgroupoid of the ordered groupoid $G$. Then the relation

$$
g \simeq_{A} h \Longleftrightarrow \text { there exist } a, b, c, d \in A \text { such that agb } \leqslant h \text { and } c h d \leqslant g
$$

is an equivalence relation on $G$. The relation

$$
[g] \leqslant[k] \Longleftrightarrow \text { there exist } a, b \in A \text { such that } a g b \leqslant k
$$

is a well-defined partial order on the set $G / / A$ of equivalence classes of $\simeq_{A}$.

Proof. It is obvious that $\simeq_{A}$ is reflexive and symmetric. Suppose that $g \simeq_{A} h$ and $h \simeq_{A} k$. Then there exist $a, b, u, v \in A$ such that $a g b \leqslant h$ and $u h v \leqslant k$. Then $a a^{-1} \leqslant h h^{-1}=u^{-1} u$ and $b^{-1} b \leqslant h^{-1} h=v v^{-1}$, and $\left(u \mid a a^{-1}\right) a g b\left(b^{-1} b \mid v\right) \leqslant$ $u h v \leqslant k$ with $\left(u \mid a a^{-1}\right) a, b\left(b^{-1} b \mid v\right) \in A$. Similarly there exist $p, q \in A$ with $p k q \leqslant g$ and $g \simeq_{A} k$.

We now show that $\leqslant$ is well-defined. Suppose that $g \simeq_{A} g^{\prime}$ and that $k \simeq_{A} k^{\prime}$. Hence there exist $p, q, u, v \in A$ with $p g^{\prime} q \leqslant g, u k v \leqslant k^{\prime}$. Now if in addition there exist $a, b \in A$ with $a g b \leqslant k$ then $\left(u \mid a a^{-1}\right) a g b\left(b^{-1} b \mid v\right) \leqslant u k v \leqslant k^{\prime}$ and

$$
\left(\left(u \mid a a^{-1}\right) a \mid p p^{-1}\right) p g^{\prime} q\left(q^{-1} q \mid b\left(b^{-1} b \mid v\right)\right) \leqslant\left(u \mid a a^{-1}\right) a g b\left(b^{-1} b v\right) .
$$

Hence $g^{\prime} \leqslant k^{\prime}$.

Now it is clear that $\leqslant$ is reflexive and transitive, and by definition of $\simeq_{A}$ it is antisymmetric.

Lemma 3.5. Let e, $f \in G_{0}$. Then $e \simeq_{A} f$ if and only if there exist $a, p \in A$ such that

$$
a a^{-1} \leqslant e, a^{-1} a=f \text { and } p p^{-1} \leqslant f, p^{-1} p=e .
$$

A pair $(a, p)$ of arrows of $A$ realizing the relation $\simeq_{A}$ for $e, f \in G_{0}$ is called an $A$-nexus between $e$ and $f$.

There is a natural ordered groupoid structure on $G / / A$ that we now begin to describe.

Lemma 3.6. Suppose that $g \simeq_{A} h$. Then $g^{-1} \simeq_{A} h^{-1}$ and $g g^{-1} \simeq_{A} h h^{-1}$.

Proof. Suppose that there exist $a, b, u, v \in A$ such that $a g b \leqslant h$ and $u h v \leqslant g$. Then $b^{-1} g^{-1} a^{-1} \leqslant h^{-1}$ and $v^{-1} h^{-1} u^{-1} \leqslant g^{-1}$. Furthermore, $(u, a)$ is an $A-$ nexus between $g g^{-1}$ and $h h^{-1}$.

When we represent relationships in $G$ diagrammatically, we shall indicate the partial order by a dashed line with the order decreasing down the page. 
Lemma 3.7. Let $g, h \in G$ with $g^{-1} g \simeq_{A} h h^{-1}$. Let $(a, p)$ be an $A-n e x u s$ between $g^{-1} g$ and $h h^{-1}$. Define

$$
\begin{aligned}
g^{\prime} & =\left(g \mid a a^{-1}\right) \\
h^{\prime} & =\left(p p^{-1} \mid h\right) \\
a^{\prime} & =\left(a \mid p p^{-1}\right) \\
p^{\prime} & =\left(p \mid a a^{-1}\right) \\
g^{\prime \prime} & =\left(g \mid a^{\prime} a^{\prime-1}\right) \\
h^{\prime \prime} & =\left(p^{\prime} p^{\prime-1} \mid h\right) .
\end{aligned}
$$

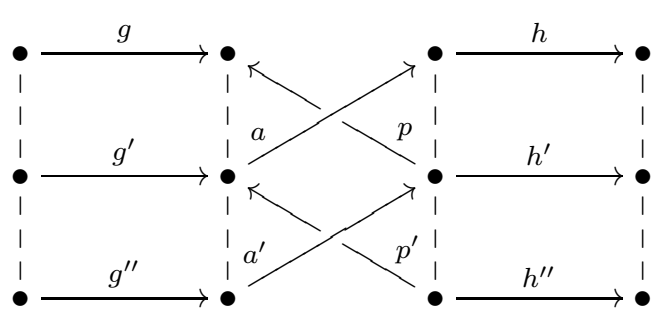

Then $g^{\prime} a h \simeq_{A} g p^{-1} h^{\prime}$, and if we make an alternative choice of nexus $\left(a_{1}, p_{1}\right)$ leading to elements $g_{1}^{\prime}, h_{1}^{\prime}$ then

$$
g_{1}^{\prime} a_{1} h \simeq_{A} g^{\prime} a h \simeq_{A} g p^{-1} h^{\prime}=g p_{1}^{-1} h_{1}^{\prime} .
$$

Proof. We have $g^{\prime} a h \geqslant g^{\prime \prime} a^{\prime} h^{\prime}=g^{\prime \prime} a^{\prime} p g^{-1} g p^{-1} h^{\prime}$ with $g^{\prime \prime} a^{\prime} p g^{-1} \in A$ by normality of $A$, and similarly $g p^{-1} h^{\prime} \geqslant g^{\prime} p^{\prime-1} h^{\prime \prime}=g^{\prime} a h h^{-1} a^{-1} p^{\prime-1} h^{\prime \prime}$ with $h^{-1} a^{-1} p^{-1} h^{\prime \prime} \in$ A.

For the alternative choice of nexus we have $a^{-1} a=a_{1}^{-1} a_{1}=h h^{-1}$ and $g_{1}^{\prime} a_{1} h \simeq_{A}$ $g_{1}^{\prime} a_{1} a^{-1} g^{\prime-1} g^{\prime} a h$ with $g_{1}^{\prime} a_{1} a^{-1} g^{\prime-1} \in A$. Hence $g_{1}^{\prime} a_{1} h \simeq_{A} g^{\prime} a h$ and similarly $g p^{-1} h^{\prime} \simeq_{A} g p_{1}^{-1} h_{1}^{\prime}$.

Given $g, h \in G$ with $g^{-1} g \simeq_{A} h h^{-1}$ we have now defined a unique class in $G / / A$ in terms of an $A$-nexus between $g^{-1} g$ and $h h^{-1}$, but independent of the $A$-nexus chosen. We temporarily denote this class by $g \gamma h \in G / / A$. Hence in the notation of Lemma 3.7

$$
g \gamma h=\left[g^{\prime} a h\right]=\left[g p^{-1} h^{\prime}\right]
$$

Lemma 3.8. Suppose that $g \simeq_{A} g_{1}$ and that $h \simeq_{A} h_{1}$. Then $g \gamma h=g_{1} \gamma h_{1}$ in $G / / A$.

Proof. Fix $h$ and choose an $A$-nexus $\left(a_{1}, p_{1}\right)$ between $g_{1}^{-1} g_{1}$ and $h h^{-1}$. Let $g_{1}^{\prime}=$ $\left(g_{1} \mid a_{1} a_{1}^{-1}\right)$ and $h_{1}^{\prime}=\left(p_{1} p_{1}^{-1} \mid h\right)$. Then $g_{1} \gamma h=\left[g_{1}^{\prime} a_{1} h\right]=\left[g_{1} p_{1}^{-1} h_{1}^{\prime}\right] \in G / / A$. 
There exist $u, v \in A$ with $u g_{1} v \leqslant g$. Let $u^{\prime}=\left(u \mid g_{1}^{\prime} g_{1}^{\prime-1}\right)$ and $v^{\prime}=\left(a_{1} a_{1}^{-1} \mid v\right)$.

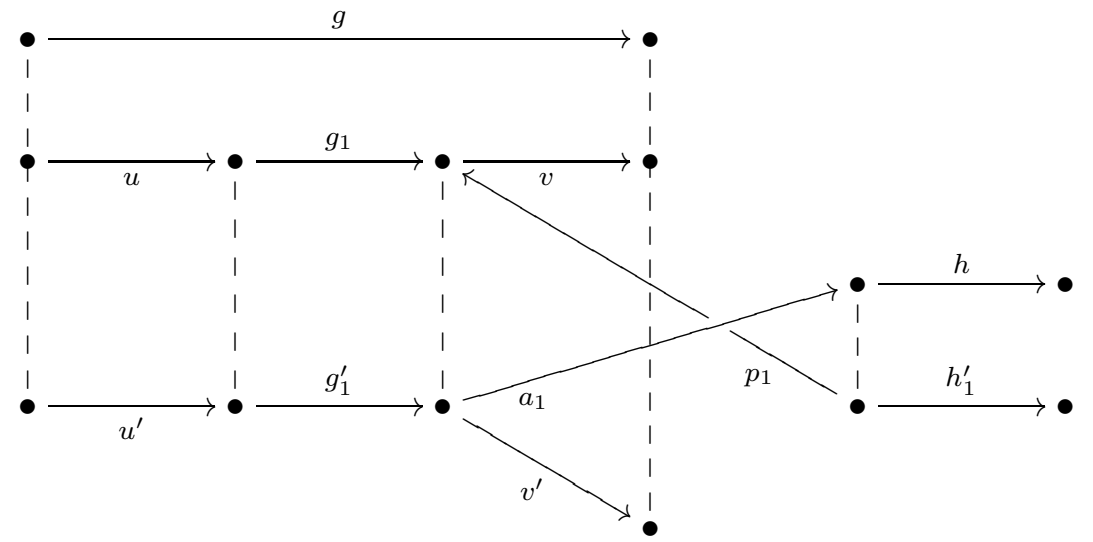

Now $v^{\prime-1} a_{1}$ is a component of an $A$-nexus between $g^{-1} g$ and $h h^{-1}$ and so can be used to define $g \gamma h$. By uniqueness of restriction we have $\left(g \mid v^{\prime-1} v^{\prime}\right)=u^{\prime} g_{1}^{\prime} v^{\prime}$ and so

$$
\begin{aligned}
g \varnothing h & =\left[u^{\prime} g_{1}^{\prime} v^{\prime} v^{\prime-1} a_{1} h\right] \\
& =\left[u^{\prime} g_{1}^{\prime} a_{1} h\right] \\
& =\left[g_{1}^{\prime} a_{1} h\right]=g_{1} \succ h .
\end{aligned}
$$

Similarly $g_{1} \varnothing h=g_{1} \gamma h_{1}$.

We now have a well-defined composition of $\simeq_{A}$-classes: $[g][h]=g \gamma h$. Suppose that $g^{-1} g \simeq_{A} h h^{-1}$ and $h^{-1} h \simeq_{A} k k^{-1}$. Choose $A$-nexuses $(a, p)$ between $g^{-1} g$ and $h h^{-1}$ and $(b, q)$ between $h^{-1} h$ and $k k^{-1}$. Then $g \gamma h=\left[g^{\prime} a h\right]$ and $g^{\prime} a h \gamma k=\left[g^{\prime} a h q^{-1} k^{\prime}\right]$. But $h \gamma k=\left[h q^{-1} k^{\prime}\right]$ and $g \gamma h q^{-1} k^{\prime}=\left[g^{\prime} a h q^{-1} k^{\prime}\right]$, and so the composition is associative. This last observation then establishes the following result.

Proposition 3.9. $G / / A$ is a groupoid under the operation of composition of $\simeq_{A^{-}}$ classes.

Lemma 3.10. Let $h \in G$ and suppose that $(a, p)$ is an $A$-nexus between $e \in G_{0}$ and $h \mathbf{d}$. Then for $h^{\prime}=(p \mathbf{d} \mid h)$, we have $\left[h^{\prime}\right]=[h]$ in $G / / A$.

Proof. Since $h^{\prime} \leqslant h$ in $G$ we have $\left[h^{\prime}\right] \leqslant[h]$. Now set $p^{\prime}=(p \mid a \mathbf{d})$ and $h^{\prime \prime}=$ $\left(p^{\prime} \mathbf{d} \mid h\right)$. Then

$$
h \simeq_{A} h\left(h^{-1} a^{-1} p^{\prime-1} h^{\prime \prime}\right)=a^{-1} p^{\prime-1} h^{\prime \prime} \simeq_{A} h^{\prime \prime} \leqslant h^{\prime}
$$

and so $[h] \leqslant\left[h^{\prime}\right]$.

We shall now proceed to verify that the partial order on $\simeq_{A}$-classes given in Lemma 3.4 makes $G / / A$ into an ordered groupoid.

Lemma 3.11. If $[g] \leqslant[h]$ in $G / / A$ then $[g]^{-1}=\left[g^{-1}\right] \leqslant\left[h^{-1}\right]=[h]^{-1}$. 
Proof. We have $a g b \leqslant h$ for some $a, b \in A$ and so $b^{-1} g^{-1} a^{-1} \leqslant h^{-1}$.

Lemma 3.12. Suppose that $\left[g_{1}\right] \leqslant\left[h_{1}\right]$ and that $\left[g_{2}\right] \leqslant\left[h_{2}\right]$ in $G / / A$ and that the compositions $\left[g_{1}\right]\left[g_{2}\right]$ and $\left[h_{1}\right]\left[h_{2}\right]$ exist. Then $\left[g_{1}\right]\left[g_{2}\right] \leqslant\left[h_{1}\right]\left[h_{2}\right]$.

Proof. There exist $a_{1}, b_{1}, a_{2}, b_{2} \in A$ such that $a_{1} g_{1} b_{1} \leqslant h_{1}$ and $a_{2} g_{2} b_{2} \leqslant h_{2}$. Since $\left[g_{i}\right]=\left[a_{i} g_{i} b_{i}\right]$ we may as well assume that $g_{i} \leqslant h_{i}$ for $i=1,2$. We have $A$-nexuses $(a, p)$ between $h_{1}^{-1} h_{1}$ and $h_{2} h_{2}^{-1}$ and $(b, q)$ between $g_{1}^{-1} g_{1}$ and $g_{2} g_{2}^{-1}$. We set $p^{\prime}=\left(p \mid g_{1}^{-1} g_{1}\right)$ and $h_{2}^{\prime \prime}=\left(p^{\prime} p^{\prime-1} \mid h_{2}^{\prime}\right)$. Then

$$
g_{1} q^{-1} g_{2}^{\prime} \simeq_{A} g_{1} q^{-1} g_{2}^{\prime} g_{2}^{\prime-1} q p^{\prime-1} h_{2}^{\prime \prime}=g_{1} p^{\prime} h_{2}^{\prime \prime}
$$

since $g_{2}^{\prime}, h_{2}^{\prime \prime} \leqslant h_{2}$ and so $g_{2}^{\prime-1} q p^{\prime-1} h_{2}^{\prime \prime} \in A$, and

$$
g_{1} p^{\prime-1} h_{2}^{\prime \prime} \leqslant h_{1} p^{-1} h_{2}^{\prime} \text {. }
$$

Therefore $\left[g_{1}\right]\left[g_{2}\right]=\left[g_{1} q^{-1} g_{2}^{\prime}\right] \leqslant\left[h_{1} p^{-1} h_{2}^{\prime}\right]=\left[h_{1}\right]\left[h_{2}\right]$.

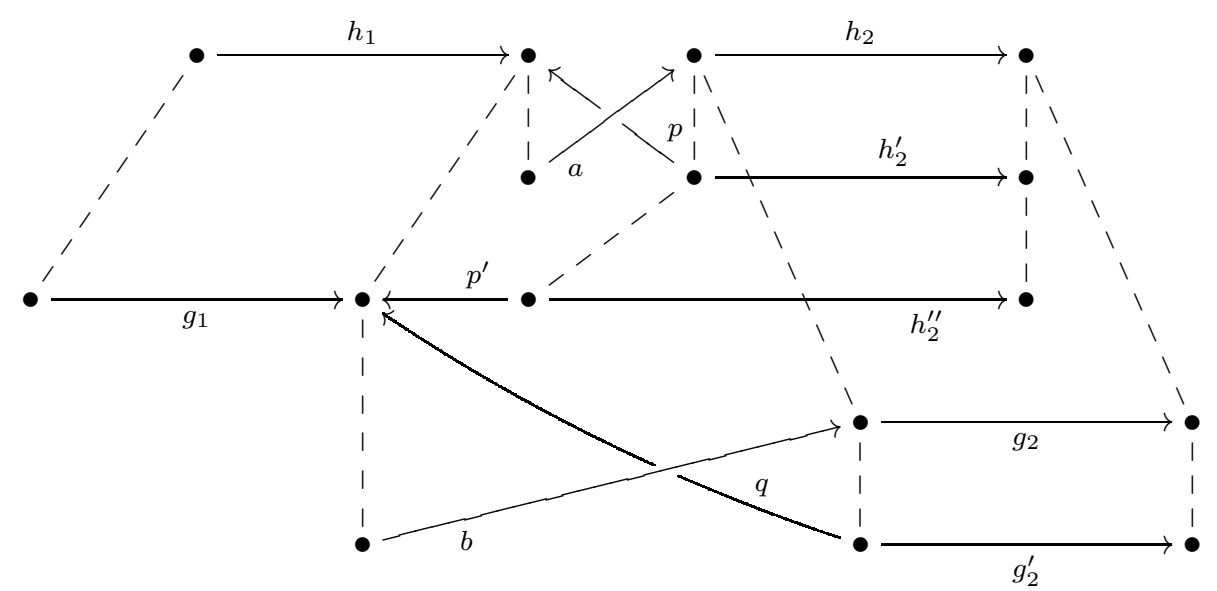

Lemma 3.13. Suppose that $[e] \leqslant\left[g g^{-1}\right]$. Then there exists a unique arrow $[k] \in$ $G / / A$ such that $[k] \leqslant[g]$ and $[k][k]^{-1}=[e]$.

Proof. Since $[e] \leqslant\left[g g^{-1}\right]$ there exists $a \in A$ with $a a^{-1} \leqslant g g^{-1}$ and $a^{-1} a=e$. Let $g^{\prime}=\left(a a^{-1} \mid g\right)$. Then $\left[g^{\prime}\right] \leqslant[g]$ and $\left[g^{\prime}\right]\left[g^{\prime}\right]^{-1}=\left[a a^{-1}\right]=[e]$.

Now suppose that $[k] \leqslant[g]$ and $[k][k]^{-1}=[e]$. We shall show that $[k]=\left[g^{\prime}\right]$. There exist $u, v \in A$ such that $u k v \leqslant g$ and an $A$-nexus $(b, q)$ between $k k^{-1}$ and $e$, so that

$$
b b^{-1} \leqslant k k^{-1}, b^{-1} b=e, q q^{-1} \leqslant e, q^{-1} q=k k^{-1} .
$$

Let $k^{\prime}=\left(b b^{-1} \mid k\right), u^{\prime}=\left(u \mid b b^{-1}\right)$ and $v^{\prime}=\left(k^{\prime-1} k^{\prime} \mid v\right)$. Then $u^{\prime} k^{\prime} v^{\prime} \leqslant u k v \leqslant g$ and

$$
g^{\prime} \simeq_{A} g^{\prime} g^{\prime-1} a b^{-1} u^{\prime-1} u^{\prime} k^{\prime} v^{\prime}
$$


since $g^{\prime} \leqslant g, u^{\prime} k^{\prime} v^{\prime} \leqslant g$, and $a b^{-1} u^{\prime-1} \in A$,

$$
=a b^{-1} k^{\prime} v^{\prime} \simeq_{A} k^{\prime} \leqslant k .
$$

Let $a^{\prime}=\left(a \mid q q^{-1}\right)$ and $g^{\prime \prime}=\left(a^{\prime} a^{\prime-1} \mid g^{\prime}\right)$.

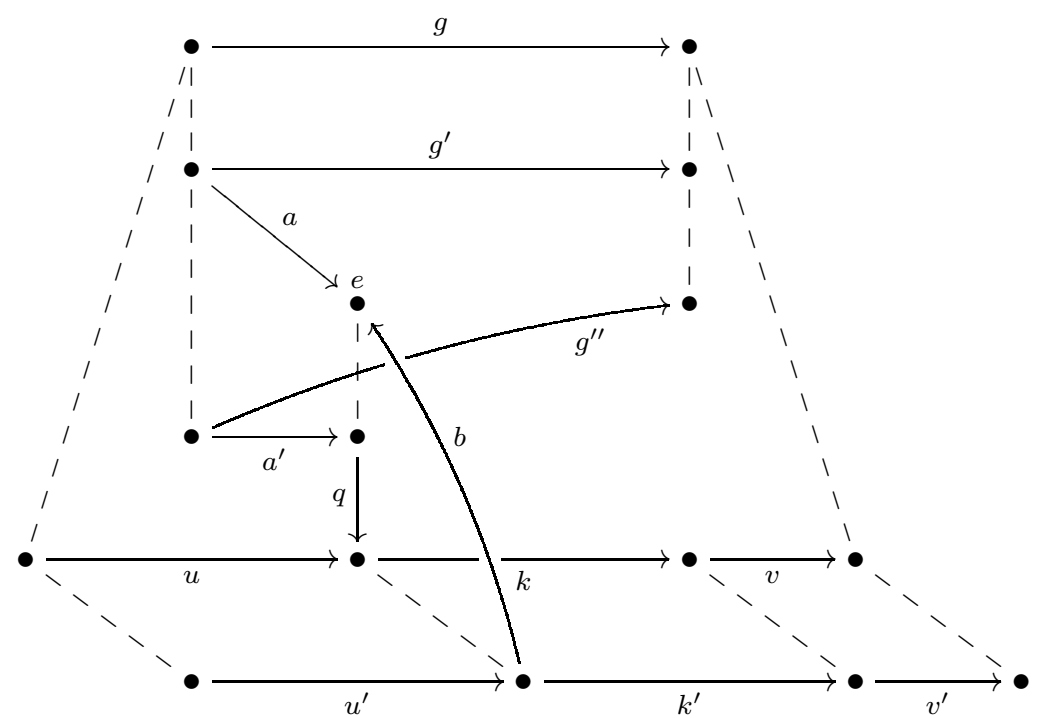

Then

$$
k \simeq_{A} k v v^{-1} k^{-1} u^{-1} u q^{-1} a^{\prime-1} g^{\prime \prime}
$$

since $u k v \leqslant g, g^{\prime \prime} \leqslant g$ and $v, u g^{-1} a^{\prime-1} \in A$,

$$
=q^{-1} a,{ }^{-1} g^{\prime \prime} \simeq g^{\prime \prime} \leqslant g^{\prime} .
$$

Hence $[k] \leqslant\left[g^{\prime}\right]$ and by symmetry $\left[g^{\prime}\right] \leqslant[k]$.

Theorem 3.14. $G / / A$ is an ordered groupoid and the quotient map $\varpi: G \rightarrow G / / A$ is a fibration.

Proof. The ordered groupoid structure follows from Proposition 3.9 and Lemmas 3.11, 3.12 and 3.13. To show that $\varpi: g \mapsto[g]$ is a fibration, consider the restriction $\operatorname{star}_{G}(e) \rightarrow \operatorname{star}_{G / / A}[e]$ and $[h] \in \operatorname{star}_{G / / A}[e]$. There exists an $A$-nexus $(a, p)$ between $e$ and $h h^{-1}$. Defining $h^{\prime}, h^{\prime \prime}, a^{\prime}, p^{\prime}$ as in Lemma 3.7.

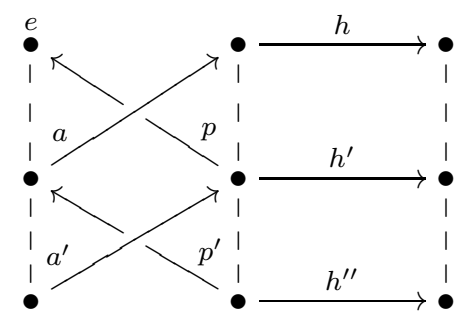


we have $\left[p^{-1} h^{\prime}\right]=\left[h^{\prime}\right]$, and $\left[h^{\prime}\right]=[h]$ by Lemma 3.10 . Hence we have $p^{-1} h^{\prime} \in$ $\operatorname{star}_{G}(e)$ and

$$
\varpi: p^{-1} h^{\prime} \mapsto\left[p^{-1} h^{\prime}\right]=\left[h^{\prime}\right]=[h] .
$$

Example 2.2, coupled with Example 3.17(iv) below shows that $\varpi$ need not be a strong fibration.

If we begin with an ordered functor $\theta: G \rightarrow H$, then by Lemma 3.2, its kernel ker $\theta$ is a normal ordered subgroupoid of $G$ and we can form the quotient ordered groupoid $G / / \operatorname{ker} \theta$. We show that $\theta$ factors through $G / / \operatorname{ker} \theta$ as the composition of the fibration $\varpi$ and a star-injective functor.

Theorem 3.15. An ordered functor $\theta: G \rightarrow H$ induces a star-injective functor $\psi: G / / \operatorname{ker} \theta \rightarrow H$ such that $\theta=\varpi \psi$.

Proof. We define $\psi: G / / \operatorname{ker} \theta \rightarrow H$ by $\psi:[g] \mapsto g \theta$. It is clear that $\psi$ is an ordered functor. Suppose that $[k] \in \operatorname{star}_{G / / \operatorname{ker} \theta}[g]$ and that $k \theta=g \theta$. Let $(a, p)$ be a $(\operatorname{ker} \theta)$-nexus between $g g^{-1}$ and $k k^{-1}$.

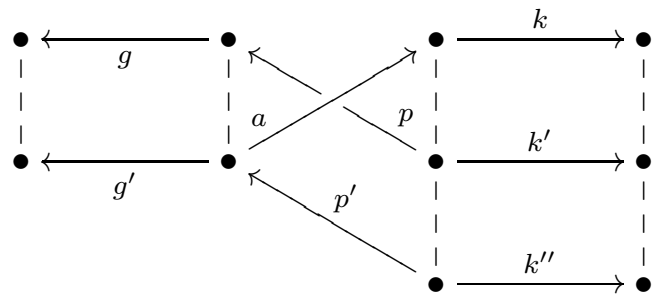

Now

$$
\left(g^{-1} p^{-1} k^{\prime}\right) \theta=(g \theta)^{-1}(p \theta)^{-1} k^{\prime} \theta=(g \theta)^{-1} k^{\prime} \theta \leqslant(g \theta)^{-1} k \theta \in H_{0} .
$$

It follows that $g^{-1} p^{-1} k^{\prime} \in \operatorname{ker} \theta$, and so

$$
k^{\prime}=p g\left(g^{-1} p^{-1} k^{\prime}\right) \simeq_{\mathrm{ker} \theta} g .
$$

But, by Lemma 3.10, we have $[k]=\left[k^{\prime}\right]$ and so $[k]=[g]$.

Corollary 3.16. If $\theta: G \rightarrow H$ is an ordered fibration then $\psi: G / / \operatorname{ker} \theta \rightarrow H$ is a covering.

Proof. In the triangle

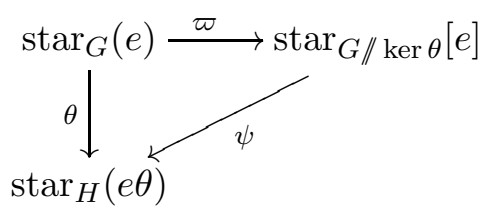

$\psi$ is star-injective by Theorem 3.15 , whilst $\theta$ and $\varpi$ are star-surjective by assumption and by Theorem 3.14 respectively. It follows that $\psi$ is star-surjective, and so $\psi$ is a covering. 
Example 3.17. (i) For a groupoid $G$, the quotient $G / G$ is isomorphic to the set $\pi_{0}(G)$ of connected components of $G$, regarded as a trivial groupoid. If $G$ is ordered, then its ordering induces a preorder on $\pi_{0}(G)$, defined as follows. If $\lfloor g\rfloor$ denotes the connected component of $g \in G$, then $\lfloor g\rfloor \leqslant\lfloor h\rfloor$ if and only if for each $h^{\prime} \in\lfloor h\rfloor$ there exists $g^{\prime} \in\lfloor g\rfloor$ with $g^{\prime} \leqslant h^{\prime}$. As a preordered set, $\pi_{0}(G)$ has a canonical partially ordered quotient $Q(G)$, and the poset $Q(G)$ is isomorphic to the ordered quotient $G / / G$.

(ii) We return to Example 2.7 For any $p \in \mathbb{N}$ and any even $a \geqslant p$ the pair $(a, 0),(p, p)$ is a $(\operatorname{ker} \theta)$-nexus between $p$ and 0 and hence $B / / \operatorname{ker} \theta$ has one object and is a group. Moreover, if $(m, n) \notin \operatorname{ker} \theta$ then $m, n$ have opposite parity and then $(m, n) \simeq_{\operatorname{ker} \theta}(0,1)$, since $(m, m)(m, n)(n, m+1)=(m, m+1) \leqslant(0,1)$ and $(2 m, 0)(0,1)(1, m+n)=(2 m, m+n) \leqslant(m, n)$. Hence $B / / \operatorname{ker} \theta$ is isomorphic to $\mathbb{Z}_{2}$.

(iii) Let $\Delta^{n}$ be the simplicial groupoid on the set $I_{n}=\{0,1, \ldots, n\}$ (see [6]): hence $\left(\Delta^{n}\right)_{0}=I_{n}$ and there is a unique arrow $(i, j)$ from $i$ to $j . \Delta^{n}$ is freely generated by the obvious directed chain $\Gamma_{n}$ with vertex set $I_{n}$. We set $\Delta=\bigsqcup_{n \geqslant 1} \Delta^{n}$. The natural partial order is trivial between elements of each $\Delta^{n}$. If $\gamma \in \Delta^{p}$ and $\delta \in \Delta^{q}$ with $p<q$ then $\gamma \geqslant \delta$ if and only if $\delta$ is the image of $\gamma$ under an embed$\operatorname{ding} \Gamma_{p} \hookrightarrow \Gamma_{q} . \Delta$ is the ordered groupoid equivalent to the monogenic free inverse semigroup $F I S(x)$.

Adjoin a minimum idempotent $z$ to $\mathscr{I}$ : we can then define a functor $\theta: \Delta \rightarrow$ $\mathscr{I} \cup\{z\}$ by mapping $\Delta^{1}$ to $\mathscr{I}$ via an isomorphism and, for all $n>1$, mapping $\delta \in \Delta^{n}$ to $z$. Then $\Delta / / \operatorname{ker} \theta$ is the ordered groupoid $\Delta^{1} \cup\left\{e_{i}: i \geqslant 2\right\}$ in which the $e_{i}$ form an infinite descending chain.

(iv) For a presheaf of groups $G=(\mathscr{G}, P)$ as in Example 1.2, an ordered normal subgroupoid is a presheaf of groups $N=(\mathscr{N}, P)$ where, for each $x \in P$, the group $N_{x}$ is a normal subgroup of $G_{x}$, and the linking maps are just the restrictions of those in $G$ so that, whenever $x \geqslant y$ in $P$, then $N_{y} \supseteq N_{x} \alpha_{y}^{x}$. Then $g \simeq_{N} h$ if and only if $g, h \in G_{x}$ for some $x$ and $g^{-1} h \in N_{x}$. Hence $G / / N$ is isomorphic to the presheaf of groups $Q=(\mathscr{Q}, P)$ where $Q_{x}=G_{x} / N_{x}$ and the linking maps for $Q$ are induced by those in $G$.

(v) Fix a group $G$ and an endomorphism $\alpha: G \rightarrow G$. The Bruck-Reilly groupoid $\operatorname{BR}(G, \alpha)$ (corresponding to the Bruck-Reilly extension of inverse semigroups introduced in [11]) determined by $(G, \alpha)$ is the ordered groupoid with object set $\mathbb{N}$, ordered by the reverse of the natural total order $\geqslant$ on $\mathbb{N}$, and arrow set $\mathbb{N} \times G \times \mathbb{N}$. For an arrow $(m, g, n)$ we have $(m, g, n) \mathbf{d}=m,(m, g, n) \mathbf{r}=n$ and the composition of arrows is $(m, g, n)(n, h, q)=(m, g h, q)$. The ordering $\succcurlyeq$ on arrows is defined as follows:

$$
(m, g, n) \succcurlyeq(p, h, q) \text { if and only if } m \leqslant p, m-n=p-q, \text { and } h=g \alpha^{p-m} .
$$


An ordered functor $\Theta: \operatorname{BR}(G, \alpha) \rightarrow \operatorname{BR}(H, \beta)$ that is the identity on objects is determined by a group homomorphism $\theta: G \rightarrow H$ satisfying $\alpha \theta=\theta \beta$, and then $\Theta:(m, g, n) \mapsto(m, g \theta, n)$. Hence

$$
\operatorname{ker} \Theta=\{(m, g, m): m \in \mathbb{N}, g \in \operatorname{ker} \theta\} .
$$

Now if $g \in \operatorname{ker} \theta$ then $g \alpha \theta=g \theta \beta=e_{H}$ and so $\alpha: \operatorname{ker} \theta \rightarrow \operatorname{ker} \theta$, and it follows that $\operatorname{ker} \Theta=\operatorname{BR}(\operatorname{ker} \theta, \alpha)$.

It is easy to see that $(m, g, n) \simeq_{\operatorname{ker} \Theta}(p, h, q)$ if and only if $m=p, n=q$ and $g \theta=h \theta$ and so we can identify $\operatorname{BR}(G, \alpha) / / \operatorname{ker} \theta$ as $\operatorname{BR}(G / \operatorname{ker} \theta, \bar{\alpha})$, where $\bar{\alpha}$ is induced by $\alpha$.

(vi) If $A$ is a normal inverse subsemigroup of an inverse semigroup $S$ then $S / / A$ need not be an inductive groupoid. Consider $S$ as shown below:

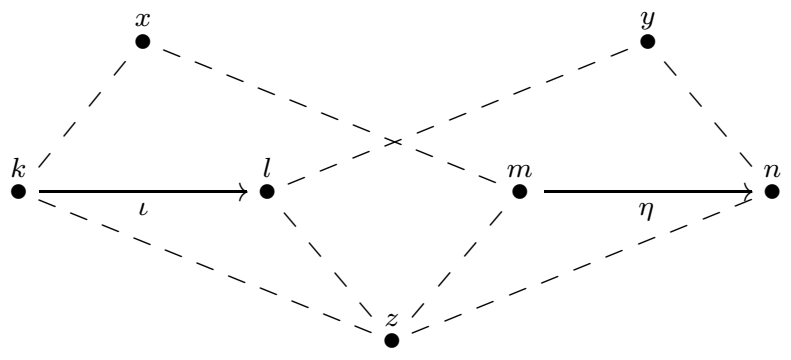

and let $A=S$. The ordered groupoid $S / / S$ is the trivial ordered groupoid

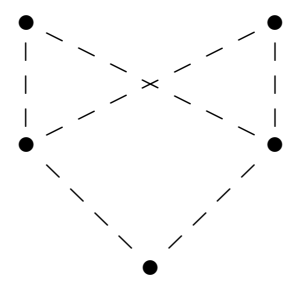

which is not inductive.

\section{THE MAXIMUM ENLARGEMENT THEOREM}

Theorem 3.15 gives a canonical factorization of an ordered groupoid morphism as a fibration followed by a star-injective functor. Ehresmann's Maximum Enlargement Theorem [4] then provides a canonical factorization of a star-injective functor as a well-structured ordered embedding - called an enlargement - followed by a covering. For an exposition of the Maximum Enlargement Theorem and its applications see [7, chapter 11]. In this section we give a short account of the Maximum Enlargement Theorem based solely on the notion of an ordered groupoid acting on a poset: this basis for a proof of the theorem was first set out in [5] and fully developed in the $\mathrm{PhD}$ thesis of the third author [10]. 
The notions of a groupoid action on a groupoid and the associated construction of the semidirect product seem to have been first defined in [2]. We describe these notions for ordered groupoids: our definitions are equivalent to those given by Steinberg [12]. An action of an ordered groupoid $G$ on an ordered groupoid $A$ is determined by the following data. We are given an ordered functor $\omega: A \rightarrow G_{0}$ and for each pair $(a, g)$ with $a \omega=g \mathbf{d}$ there exists an element $a \triangleleft g \in A$ such that

- $(a \triangleleft g) \omega=g \mathbf{r}$,

- if $g, h \in G$ are two composable arrows and if $a \in A$ satisfies $a \omega=g \mathbf{d}$, then $a \triangleleft g h=(a \triangleleft g) \triangleleft h$,

- if $a, b \in A$ are two composable arrows and if $g \in G$ satisfies $a \omega=g \mathbf{d}=$ $b \omega$, then $a \triangleleft g$ and $b \triangleleft g$ are composable, and $(a b) \triangleleft g=(a \triangleleft g)(b \triangleleft g)$,

- $a \triangleleft a \omega=a$,

- if $a \leqslant b$ in $A$ and $g \leqslant h \in G$ with $a \omega=g \mathbf{d}$ and $b \omega=h \mathbf{d}$ then $a \triangleleft g \leqslant b \triangleleft h$ in $A$.

This definition includes that of an ordered groupoid $G$ acting on a poset $P$, where $P$ is considered as a trivial groupoid.

Given an action of the ordered groupoid $G$ on the ordered groupoid $A$ the semidirect product or action groupoid $G \ltimes A$ was defined by Brown [2] in the unordered case, and by Steinberg [12] for ordered groupoids. $G \ltimes A$ is an ordered groupoid whose set of arrows is the pullback $\{(g, a): a \omega=g \mathbf{r}\}$. The domain and range maps are defined by

$$
(g, a) \mathbf{d}=\left(g \mathbf{d},\left(a \triangleleft g^{-1}\right) \mathbf{d}\right) \text { and }(g, a) \mathbf{r}=(g \mathbf{r}, a \mathbf{r})
$$

and the composition is

$$
(g, a)(h, b)=(g h,(a \triangleleft h) b)
$$

defined when $g \mathbf{r}=h \mathbf{d}$ and $a \mathbf{r}=\left(b \triangleleft h^{-1}\right) \mathbf{d}$. The ordering is componentwise. We note that we can identify $(G \ltimes A)_{0}$ with $A_{0}$ since if $(x, y) \in(G \ltimes A)_{0}$ then $x=y \omega$.

Lemma 4.1. The projection map $\pi:(g, a) \mapsto g$ is a strong fibration $G \ltimes A \rightarrow G$.

Proof. Given a commutative square

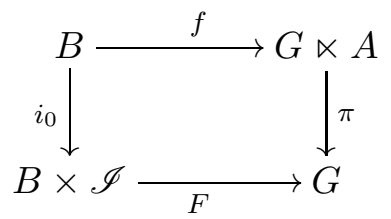

in which $b f=\left(g_{b}, a_{b}\right) \in G \ltimes A$ and $(y, \iota) F=h_{y} \in G$, we define

$$
(b, \iota) \widetilde{F}=\left(g_{b} h_{b \mathbf{r}}, a_{b} \triangleleft h_{b \mathbf{r}}\right) .
$$

This is an ordered functor lifting $F$. 
4.1. Actions on posets and coverings of ordered groupoids. There is an equivalence of categories between the category of coverings of a groupoid $G$ and the category of actions of $G$ on sets. This idea originates with Ehresmann [4] but is discussed explicitly in [6, Proposition 30] and in [3, Proposition 1.2]. To an action of $G$ on the set $X$ there corresponds the projection $\pi: G \ltimes X \rightarrow G$, and given any covering $C \rightarrow G$ there is an action of $G$ on $C_{0}$. This equivalence generalizes to one for ordered groupoids acting on posets. The details of this generalisation were set out in [10], and we record the essentials here.

Let $\gamma: C \rightarrow G$ be an ordered covering of groupoids. We define an action of $G$ on $C_{0}$ as follows. The covering $\gamma$ restricts to an ordered mapping $\gamma: C_{0} \rightarrow G_{0}$, and if $x \gamma=g \mathbf{d}$ there exists a unique $c \in \operatorname{star}_{C}(x)$ with $c \gamma=g$ : we define $a \triangleleft g=c \mathbf{r}$. The projection map $\pi: G \ltimes C_{0} \rightarrow G$ is then a covering naturally isomorphic to $C \rightarrow G$. On the other hand, given an action of $G$ on a poset $P$, the action groupoid $G \ltimes P$ has $(G \ltimes P)_{0}=P$ and the action of $G$ on $P$ derived from the covering $G \ltimes P \rightarrow G$ is the given action of $P$ on $G$.

For a fixed ordered groupoid $G$, let $\operatorname{Act}(G)$ be the category whose objects are $G$-posets and whose morphisms are maps of $G$-posets, and let $\operatorname{Cov}(G)$ be the category whose objects are ordered coverings $C \rightarrow G$ and in which a morphism from $\gamma: C \rightarrow G$ to $\delta: C^{\prime} \rightarrow G$ is an ordered functor $\phi: C \rightarrow C^{\prime}$ such that $\phi \delta=\gamma$. Then the correspondences between coverings and poset actions just outlined give the following result.

Proposition 4.2. For a fixed ordered groupoid $G$, the categories $\operatorname{Act}(G)$ and $\operatorname{Cov}(G)$ are naturally equivalent.

4.2. Constructing a covering from an immersion. Let $\phi: U \rightarrow H$ be an immersion of ordered groupoids. Our aim is to factorize $\phi$ through a covering $\widetilde{H}_{\phi} \stackrel{\pi}{\rightarrow} H$, and by Proposition 4.2 this amounts to constructing an $H$-action on a suitable poset. We may regard the set of arrows of $H$ as a poset, which we denote by $\Omega H$ : we justify this notation in section 5 (We note that $\Omega H$ is denoted by $H_{D}$ in [12].) We now construct the free right $H$-poset on $U_{0}$ : this is the pullback

$$
U_{0} \otimes \Omega H=\{(e, h): e \phi=h \mathbf{d}\}
$$

ordered componentwise, and there is an obvious right $H$-action by multiplication, so we can form the semidirect product

$$
H \ltimes\left(U_{0} \otimes \Omega H\right)=\{(k,(e, h)): e \phi=h \mathbf{d}, h \mathbf{r}=k \mathbf{r}\} .
$$

As above, we identify the object set of the semidirect product with $U_{0} \otimes H$ : then we have

$$
(k,(e, h)) \mathbf{d}=\left(e, h k^{-1}\right) \text { and }(k,(e, h)) \mathbf{r}=(e, h)
$$

and the composition of $(k,(e, h))$ and $(m(f, l))$, defined when $(e, h)=\left(f, l m^{-1}\right)$, is given by

$$
(k,(e, h))(m,(f, l))=(k m,(f, l)) .
$$

We now wish to define an ordered embedding $\iota: U \rightarrow H \ltimes\left(U_{0} \otimes \Omega H\right)$, but the obvious candidate mapping $u \mapsto(u \phi,(u \mathbf{d}, u \phi))$ is not a functor: $(u \phi,(u \mathbf{d}, u \phi)) \mathbf{r}=$ 
$(u \mathbf{d}, u \phi)$ whilst $(u \mathbf{r}) \iota=(u \mathbf{r}, u \mathbf{r} \phi)$. Similar problems arise with variations upon this idea. The remedy is to impose an equivalence relation $\equiv_{U}$ on $U_{0} \otimes \Omega H$ :

$$
(e, h) \equiv_{U}(f, k) \Longleftrightarrow \text { there exists } u \in U(e, f) \text { such that } h=(u \phi) k .
$$

It is clear that $\equiv_{U}$ is an equivalence relation, and that for all $u \in U,(u \mathbf{d}, u \phi) \equiv_{U}$ $(u \mathbf{r}, u \mathbf{r} \phi)$. We denote the equivalence class of $(e, h)$ by $e \otimes h$ and the quotient set $\left(U_{0} \otimes \Omega H\right) / \equiv_{U}$ by $U_{0} \otimes \Omega H$.

Lemma 4.3. The quotient set $U_{0} \otimes \Omega H$ is a poset on which $H$ acts on the right.

Proof. The ordering on $U_{0} \otimes \Omega H$ is induced from the componentwise ordering on the pullback $U_{0} \otimes \Omega H$ : we have

$$
f \otimes h \leqslant e \otimes k \Longleftrightarrow f \otimes h=x \otimes l \text { with } x \leqslant e \text { and } l \leqslant k .
$$

We show that this is a well-defined relation on $U_{0} \otimes \Omega H$. If $f \otimes h \leqslant e \otimes k$ as above, then there exists $v \in U(f, x)$ such that $h=(v \phi) l$, and if $e \otimes k=e^{\prime} \otimes k^{\prime}$ then there exists $u \in U\left(e, e^{\prime}\right)$ such that $k=(u \phi) k^{\prime}$. Set $y=(x \mid u) \mathbf{r}$ and $k^{\prime \prime}=\left(y \phi \mid k^{\prime}\right)$. Then $(x \phi \mid u \phi) k^{\prime \prime} \leqslant(u \phi) k^{\prime}=k$ and $(x \phi \mid u \phi) \mathbf{d}=x \phi=l \mathbf{d}$. But $l \leqslant k$, and so by uniqueness of restriction, we have $l=(x \phi \mid u \phi) k^{\prime \prime}$. Now

$$
\begin{aligned}
e \otimes k=e^{\prime} \otimes k^{\prime} & \geqslant y \otimes k^{\prime \prime}=f \otimes(v \phi)(x \mid u) \phi k^{\prime \prime} \\
& =f \otimes(v \phi)(x \phi \mid u \phi) k^{\prime \prime} \\
& =f \otimes(v \phi) l=f \otimes h .
\end{aligned}
$$

It is now clear that $\leqslant$ is reflexive and transitive on $U_{0} \otimes H$. To show that it is anti-symmetric, suppose that $e \otimes k \leqslant f \otimes h \leqslant e \otimes k$. Then there exist $x \leqslant f$ and $u \in U(x, e)$ such that $(u \phi) k \leqslant h$, and $e \otimes k=x \otimes(u \phi) k$. Hence we may as well assume that $e \leqslant f$ and $k \leqslant h$. Since $F \otimes h \leqslant e \otimes k$ there exist $y \leqslant e$ and $v \in U(y, f)$ such that $(v \phi) h \leqslant k$. So

$$
y \leqslant e \leqslant f \text { and }(v \varphi) h \leqslant k \leqslant h .
$$

Since $(v(\phi) h) \mathbf{r}=h \mathbf{r}$ we deduce that $(v \phi) h=h$ and so $v \phi=h \mathbf{d}=f \phi$. Since $\phi$ is star-injective, $v=f$ and so $h=k, y=f$ and so finally $e=f$.

The right action of $H$ on $U_{0} \otimes \Omega H$ is defined by right multiplication: if $f \otimes h=x \otimes l$ then $f \mathbf{r}=l \mathbf{r}$ and so for $m \in H$ we may define $(f \otimes h) \triangleleft m=f \otimes h m$.

We now form the semidirect product $\widetilde{H}_{\phi}=H \ltimes\left(U_{0} \otimes \Omega H\right)$. We have an ordered embedding $i: U \rightarrow \widetilde{H}_{\phi}$ mapping $u \mapsto(u \phi, u \mathbf{d} \otimes u \phi)$ and a covering $\pi: \widetilde{H}_{\phi} \rightarrow H$ such that $\phi=i \pi$. In fact, $i$ has additional properties. An enlargement of ordered groupoids is an inclusion of a subgroupoid $A \hookrightarrow B$ such that

- $A_{0}$ is an order ideal in $B_{0}$,

- if $b \in B$ and $b \mathbf{d}, b \mathbf{r} \in A$ then $b \in A$,

- if $e \in B_{0}$ then there exists $b \in B$ with $b \mathbf{d}=e$ and $b \mathbf{r} \in A_{0}$. 
Theorem 4.4 (Maximum enlargement [4, 7]). Let $\phi: U \rightarrow H$ be a star-injective ordered functor. The semidirect product $\widetilde{H}_{\phi}$ is an enlargement of $U$, such that for any factorization of $\phi$ as the composition $U \stackrel{j}{\rightarrow} C \stackrel{\xi}{\rightarrow} H$ of an ordered embedding and an ordered covering, there exists a unique ordered functor $\nu: \widetilde{H}_{\phi} \rightarrow C$ such that $j=i \nu$ and $\pi=\nu \xi$.

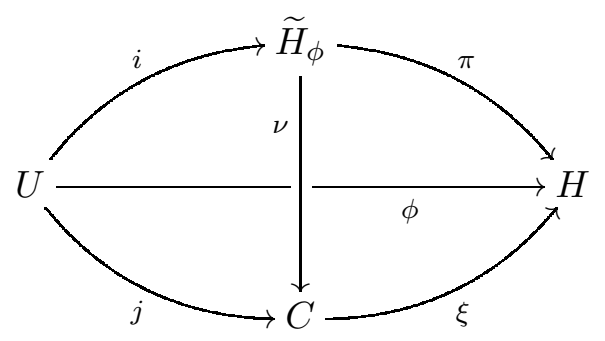

Proof. We first show that $\widetilde{H}_{\phi}$ is an enlargement of $U i$. To show that $(U i)_{0}$ is an order ideal in $\left(\widetilde{H}_{\phi}\right)_{0}$, suppose that $f \otimes h \leqslant u \mathbf{d} \otimes u \phi$. Then $f \otimes h=x \otimes l$ with $x \leqslant u \mathbf{d}$ and $l \leqslant u \phi$. Since $x \phi=l \mathbf{d}$ it follows that $l=(x \phi \mid u \phi)=(x \mid u) \phi$ and hence that $f \otimes h=x \otimes(x \mid u) \phi \in(U i)_{0}$.

Now suppose that $e \otimes k=(h, e \otimes k) \mathbf{d}=u \mathbf{d} \otimes u \phi$ and that $e \otimes k h^{-1}=(h, e \otimes k) \mathbf{r}=$ $v \mathbf{d} \otimes v \phi$ for some $u, v \in U$. Hence there exist $a \in U(u \mathbf{d}, e)$ such that $u \phi=(a \phi) k$ and $b \in U(v \mathbf{d}, e)$ such that $v \phi=(b \phi) k h^{-1}$. Then

$$
h=(v \phi)^{-1}(b \phi) k=(v \phi)^{-1}(b \phi)(a \phi)^{-1}(u \phi)=\left(v^{-1} b a^{-1} u\right) \phi .
$$

Now $i: v^{-1} b a^{-1} u \mapsto(h, v \mathbf{r} \otimes h)$, but

$$
v \mathbf{r} \otimes h=v \mathbf{r} \otimes\left(v^{-1} b a^{-1} u\right) \phi=e \otimes\left(a^{-1} u\right) \phi=e \otimes k
$$

and so $(h, e \otimes k) \in U i$.

Now given $e \otimes k \in\left(\widetilde{H}_{\phi}\right)_{0}$ the arrow $\left(k^{-1}, e \otimes k \mathbf{d}\right)$ has domain $e \otimes k$ and range $e \otimes k \mathbf{d}=e \otimes e \phi \in(U i)_{0}$.

This completes the verification that $\widetilde{H}_{\phi}$ is an enlargement of $U i$.

Now suppose that we are given a factorization of $\phi$ as the composition $U \stackrel{j}{\rightarrow} C \stackrel{\xi}{\rightarrow}$ $H$ of an ordered embedding and an ordered covering. For $(h, e \otimes k) \in \widetilde{H}_{\phi}$ we have $k \in \operatorname{star}_{H}(e \phi)=\operatorname{star}_{H}(e j \xi)$, and since $\xi$ is a covering there exists a unique $c \in \operatorname{star}_{C}(e j)$ with $c \xi=k$. Let $y=c \mathbf{r}$ : then $y \xi=k \mathbf{r}=h \mathbf{r}$ and so there exists a unique $q \in C$ with $q^{-1} \in \operatorname{star}_{C}(y)$ and $q \xi=h$. We define $(h, e \otimes k) \nu=q$.

This is well-defined since, if $e \otimes k=f \otimes l$ then there exists $u \in U(e, f)$ with $(u \phi) l=k$. Then the unique element of $\operatorname{star}_{C}(x)$ mapping to $l$ is $(u j)^{-1} c$ and we obtain $y=c \mathbf{r}=\left((u j)^{-1} c\right) \mathbf{r}$ and hence $q$ as before.

To show that $\nu$ is a functor, consider a composition $(h, e \otimes k)(l, e \otimes k l)=(h l, e \otimes$ $k l) \in \widetilde{H}_{\phi}$, with $c, y, q$ defined as above, and $(H, e \otimes k) \nu=q$. There exists a 
unique $d \in \operatorname{star}_{C}(y)$ with $d \xi=l$. Then $(l, e \otimes k l) \nu=d$ and $(h l, e \otimes k l)=q d$, and so $\nu$ is a functor. If $(h, e \otimes k) \leqslant(l, f \otimes m)$ then we have $h \leqslant l$ and we may assume that $e \leqslant f$ and $k \leqslant m$. We find a unique $s \in \operatorname{star}_{C}(f j)$ with $s \xi=m$. Then $(e j \mid s) \xi \mathbf{d}=e \phi$ and $(e j \mid s) \xi \leqslant s \xi=m$ : hence $(e j \mid s)=c$. Now there exists $t^{-1} i n \operatorname{star}_{C}(s \mathbf{r})$ with $t \xi=l$ and $(l, f \otimes m) \nu=t$. Since $c \leqslant s$ we have $y \leqslant s \mathbf{r}$ and $(t \mid y) \xi \leqslant l$. Hence $(t \mid y) \xi=h$ and so $q=(t \mid y) \leqslant t$. Hence $\nu$ is an ordered functor.

To show that $\nu$ is unique with the stated properties, suppose that $\mu$ also possesses them. Then for $(h, e \otimes k) \in \widetilde{H}_{\phi}$, we have

$$
h=(h, e \otimes k) \pi=(h, e \otimes k) \nu \xi=(h, e \otimes k) \mu \xi
$$

and since $\xi$ is a covering, if $\mu$ and $\nu$ agree on the identity $e \otimes k h^{-1}$ then $(h, e \otimes k) \nu=$ $(h, e \otimes k) \mu$. But $\mu$ and $\nu$ agree on $\left(\widetilde{H}_{\phi}\right)_{0}$ : for since $\iota \nu=j=\iota \mu$ we have that $\mu=\nu$ on $U \iota$, and then for any $z \in\left(\widetilde{H}_{\phi}\right)_{0}$ we can join $z$ to an $x \in(U \iota)_{0}$ by some $a \in \widetilde{H}_{\phi}$. But then $a \mu=a \nu$ and so $z \mu=z \nu$.

Corollary 4.5. Any ordered functor $\phi: G \rightarrow H$ admits a canonical factorization as the composition of a fibration, followed by an enlargement, followed by a covering.

$$
G \rightarrow G / / \operatorname{ker} \phi \rightarrow \widetilde{H}_{\psi} \rightarrow H
$$

\section{THE FIBRATION THEOREM AND THE DERIVED ORDERED GROUPOID}

In [12] Steinberg introduced a construction that he called the derived ordered groupoid $\operatorname{Der}(\phi)$ of a mapping $\phi: G \rightarrow H$ of ordered groupoids. Amongst its applications is the Fibration Theorem [12, Theorem 5.1]: any mapping of ordered groupoids can be factorized as the composition of an enlargement followed by a fibration. The following approach to $\operatorname{Der}(\phi)$ is based on a construction from topology - the mapping cocylinder - that is used to prove a similar theorem: every continuous map of topological spaces can be factorised as the composition of a homotopy equivalence followed by a fibration (see for example [14, Theorem I.7.30], where the construction is called the mapping path space.). Steinberg constructs the mapping cocylinder indirectly, as a semidirect product $H \ltimes \operatorname{Der}(\phi)$. We give a direct account of Steinberg's factorization, following but slightly simplifying the formulation in [8].

We start with the mapping groupoid $\operatorname{OGPD}(\mathscr{I}, H)$. Recall that this is a groupoid whose objects are the groupoid maps $\mathscr{I} \rightarrow H$ : however, any such map is completely determined by the image of the arrow $\iota$ of $\mathscr{I}$, and so we may identify the objects of $\operatorname{OGPD}(\mathscr{I}, H)$ with the poset $\Omega H$ of arrows of $H$. An arrow in $\operatorname{OGPD}(\mathscr{I}, H)$ is a natural transformation between maps $\mathscr{I} \rightarrow H$. Identifying two such maps with arrows $h_{0}, h_{1} \in \Omega H$, a natural transformation from $h_{0}$ to $h_{1}$ is 
then a pair of arrows $\left(t_{0}, t_{1}\right)$ of $H$ such that the square

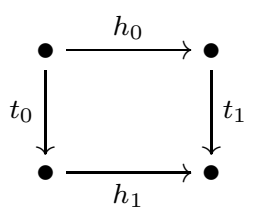

commutes. Now any three of the arrows in the square determines the fourth. We choose to suppress $t_{1}$ : rewriting $t_{0}$ as $t$, the arrow in $\operatorname{OGPD}(\mathscr{I}, H)$ in (5.1) will be identified with the triple $\left[h_{0}, t, h_{1}\right]$ of arrows in $H$, which are subject to the condition that the composition $h_{0}^{-1} t h_{1}$ exists. Then we have

$$
\left[h_{0}, t, h_{1}\right] \mathbf{d}=h_{0},\left[h_{0}, t, h_{1}\right] \mathbf{r}=h_{1},
$$

and the composition of $\left[h_{0}, t, h_{1}\right]$ and $\left[h_{1}, u, s_{1}\right]$ is defined to be

$$
\left[h_{0}, t, h_{1}\right]\left[h_{1}, u, s_{1}\right]=\left[h_{0}, t u, s_{1}\right] .
$$

Proposition 5.1. For $i=1,2$ the ordered functors $\varepsilon_{i}: \operatorname{OGPD}(\mathscr{I}, H) \rightarrow H$ given by

$$
\varepsilon_{0}:\left\{\begin{array}{l}
h \mapsto h \mathbf{d} \\
{\left[h_{0}, t, h_{1}\right] \mapsto t}
\end{array} \quad \text { if } h \in \Omega H\right.
$$

and

$$
\varepsilon_{1}:\left\{\begin{array}{l}
h \mapsto h \mathbf{r} \\
{\left[h_{0}, t, h_{1}\right] \mapsto h_{o}^{-1} t h_{1}}
\end{array} \quad \text { if } h \in \Omega H\right.
$$

are strong fibrations.

Proof. We first consider $\varepsilon_{0}$. Suppose that, in a commutative square

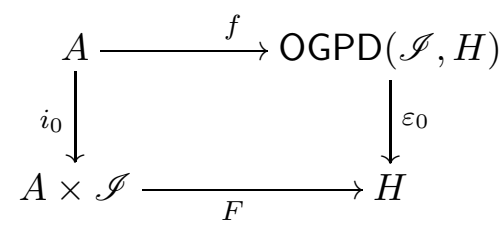

we have, for $x \in A_{0}, x f=h_{x} \in \Omega H$. and that if $a \in A$ with $a \mathbf{d}=x$ and $a \mathbf{r}=y$ then $a f=\left[h_{x}, t_{a}, h_{y}\right]$. Suppose further that $(y, \iota) F=l_{y}$. Then

$$
l_{y} \mathbf{d}=(y, \iota) F \mathbf{d}=(y, \iota) \mathbf{d} F=(y, 0) F=y f \varepsilon_{0}=h_{y} \mathbf{d}=t_{a} \mathbf{r} .
$$

Define $\widetilde{F_{0}}$ by:

$$
\widetilde{F_{0}}:\left\{\begin{aligned}
(x, 0) & \mapsto h_{x} \\
(y, 1) & \mapsto l_{y} \mathbf{r} \\
(a, \iota) & \mapsto\left[h_{x}, t_{a} l_{y}, l_{y} \mathbf{r}\right] \\
& 24
\end{aligned}\right.
$$


Certainly this makes

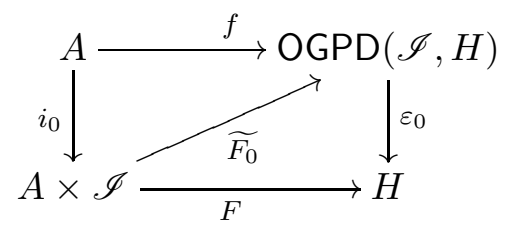

commute, and it is easy to check that this defines an ordered functor.

The argument for $\varepsilon_{1}$ is similar. In a commutative square

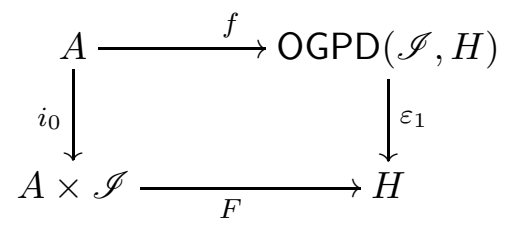

with af $=\left[h_{x}, t_{a}, h_{y}\right]$ as above, and with $(y, \iota) F=l_{y}$, we have

$$
l_{y} \mathbf{d}=(y, \iota) F \mathbf{d}=(y, \iota) \mathbf{d} F=(y, 0) F=y f \varepsilon_{1}=h_{y} \mathbf{r} .
$$

leading to the definition of a homotopy lifting $\widetilde{F_{1}}: A \times \mathscr{I} \rightarrow \operatorname{OGPD}(\mathscr{I}, H)$ by $(a, \iota) \widetilde{F_{1}}=\left[h_{x}, t_{a}, h_{y} l_{y}\right]$.

Given $\phi: G \rightarrow H$ its mapping cocylinder $M^{\phi}$ is the pullback of the diagram

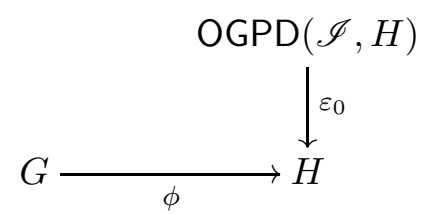

that is

$$
M^{\phi}=\left\{\left(a,\left[h_{0}, a \phi, h_{1}\right]\right): a \in G, h_{0}, h_{1} \in H, h_{0}^{-1}(a \phi) h_{1} \text { exists }\right\} .
$$

We abbreviate the 4 -tuple $\left(a,\left[h_{0}, a \phi, h_{1}\right]\right)$ to the triple $\left\langle h_{0}, a, h_{1}\right\rangle$ so that now

$$
M^{\phi}=\left\{\left\langle h_{0}, a, h_{1}\right\rangle: a \in G, h_{0}, h_{1} \in H, h_{0}^{-1}(a \phi) h_{1} \text { exists }\right\} .
$$

Then $M^{\phi}$ is an ordered groupoid with $M_{0}^{\phi}$ equal to the pullback

$$
\left\{(e, h) \in G_{0} \times H: e \phi=h \mathbf{d}\right\} .
$$

We have

$$
\left\langle h_{0}, a, h_{1}\right\rangle \mathbf{d}=\left(a \mathbf{d}, h_{0}\right),\left\langle h_{0}, a, h_{1}\right\rangle \mathbf{r}=\left(a \mathbf{r}, h_{1}\right)
$$

and if $\left(a \mathbf{r}, h_{1}\right)=\left(b \mathbf{d}, k_{0}\right)$ then the composition of $\left\langle h_{0}, a, h_{1}\right\rangle$ and $\left\langle k_{0}, b, k_{1}\right\rangle$ is given by

$$
\left\langle h_{0}, a, h_{1}\right\rangle\left\langle k_{0}, b, k_{1}\right\rangle=\left\langle h_{0}, a b, k_{1}\right\rangle .
$$

The ordering on arrows in $M^{\phi}$ is componentwise:

$$
\left\langle h_{0}, a, h_{1}\right\rangle \leqslant\left\langle k_{0}, b, k_{1}\right\rangle \Longleftrightarrow h_{25} h_{0} \leqslant k_{0}, a \leqslant b \text {, and } h_{1} \leqslant k_{1} .
$$


We can now complete our account of Steinberg's Fibration Theorem [12, Theorem 5.1], which follows that given in [8, Theorem 4.9].

Theorem 5.2. Let $\phi: G \rightarrow H$ be a morphism of ordered groupoids. Then $\phi$ admits a factorization

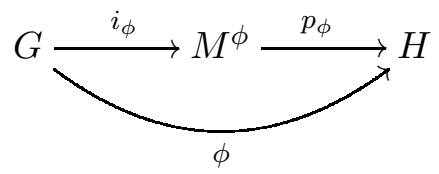

where $i_{\phi}: g \mapsto\langle(g \mathbf{d}) \phi, g,(g \mathbf{r}) \phi\rangle$ and $M^{\phi}$ is an enlargement of $G i_{\phi}$, and $p_{\phi}$ : $\left\langle h_{0}, a, h_{1}\right\rangle \mapsto h_{0}^{-1}(a \phi) h_{1}$ is a strong fibration.

Proof. It is clear that $i_{\phi}$ and $p_{\phi}$ are morphisms of ordered groupoids and that $\phi=$ $i_{\phi} p_{\phi}$.

Suppose we are given a commutative square

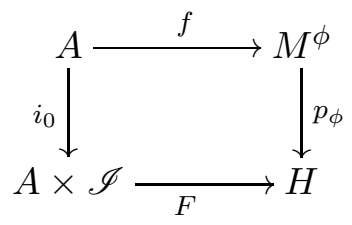

For an arrow $a \in A$, let $a f=\left\langle h_{a}, g_{a}, k_{a}\right\rangle \in M^{\phi}$ so that $(a, 0) F=h_{a}^{-1}\left(g_{a} \phi\right) k_{a}$. Suppose that $(a \mathbf{r}, \iota) F=l_{a \mathbf{r}} \in H$. Then

$$
l_{a \mathbf{r}} \mathbf{d}=(a \mathbf{r}, \iota) F \mathbf{d}=(a \mathbf{r}, \iota) \mathbf{d} F=(a \mathbf{r}, 0) F=(a \mathbf{r}) f p_{\phi}=\left(a f p_{\phi}\right) \mathbf{r}=k_{a} \mathbf{r} .
$$

Hence $k_{a}$ and $l_{a \mathbf{r}}$ are composable arrows in $H$, and we define

$$
(a, \iota) \widetilde{F}=\left\langle h_{a}, g_{a}, k_{a} l_{a \mathbf{r}}\right\rangle .
$$

This is an ordered groupoid morphism that lifts $F$.

Now $G i_{\phi}=\{\langle(g \mathbf{d}) \phi, g,(g \mathbf{r}) \phi\rangle: g \in G\}$ is a subgroupoid of $M^{\phi}$, with $\left(G i_{\phi}\right)_{0}=$ $\left\{(e, e \phi): e \in G_{0}\right\}$. Suppose that $(x, h) \in M_{0}^{\phi}$ and that $(x, h) \leqslant(e, e \phi)$. Then $x \phi=h \mathbf{d}, x \leqslant e$ and $h \leqslant e \phi$. The last condition implies that $h \in H_{0}$, whence $h=x \phi$ and so $(x, h) \in\left(G i_{\phi}\right)_{0}$. We have shown that $\left(G i_{\phi}\right)_{0}$ is an order ideal in $M_{0}^{\phi}$.

Now suppose that $\left\langle h_{0}, a, h_{1}\right\rangle \in M^{\phi}$ and that $\left(a \mathbf{d}, h_{0}\right)=(x, x \phi)$ and $\left(a \mathbf{r}, h_{1}\right)=$ $(y, y \phi)$ with $x, y \in G_{0}$. Then $\left\langle h_{0}, a, h_{1}\right\rangle=\langle(a \mathbf{d}) \phi, a,(a \mathbf{r}) \phi\rangle \in G i_{\phi}$, which establishes the second condition for an enlargement. Finally, take $(e, h) \in M_{0}^{\phi}$. Then $\langle h, e, e \phi\rangle \mathbf{d}=(e, h)$ and $\langle h, e, e \phi\rangle \mathbf{r}=(e, e \phi) \in\left(G i_{\phi}\right)_{0} .$. This concludes the proof that $M^{\phi}$ is an enlargement of $G i_{\phi}$. 
Corollary 5.3. Let $\phi: G \rightarrow H$ be a morphism of ordered groupoids. Then $\phi$ admits a factorization

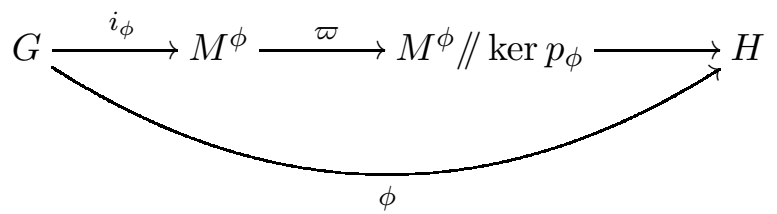

as an enlargement followed by a strong fibration followed by a covering.

Proof. Use Corollary 3.16 to factorize $p_{\phi}$ through $M^{\phi} / / \operatorname{ker} p_{\phi}$. The quotient map $\varpi: M^{\phi} \rightarrow M^{\phi} / / \operatorname{ker} p_{\phi}$ is a strong fibration by Proposition 2.4.

Corollary 5.4. The kernel of the fibration $p_{\phi}$ is equal to Steinberg's derived ordered groupoid Der $(\phi)$.

Proof. We have

$$
\begin{aligned}
\operatorname{ker} p_{\phi} & =\left\{\left\langle h_{0}, a, h_{1}\right\rangle: h_{0}^{-1}(a \phi) h_{1} \in H_{0}\right\} \\
& =\left\{\left\langle h_{0}, a, h_{1}\right\rangle: h_{0}=(a \phi) h_{1}\right\} .
\end{aligned}
$$

We can suppress mention of $h_{0}$ in elements of $\operatorname{ker} p_{\phi}$, and so write $\operatorname{ker} p_{\phi}=$ $\{(a, h):(a \phi) \mathbf{r}=h \mathbf{d}\}$. In this notation $\left(\operatorname{ker} p_{\phi}\right)_{0}=\{(e, h): e \phi=h \mathbf{d}\}$ with $(a, h) \mathbf{d}=(a \mathbf{d},(a \phi) h)$ and $(a, h) \mathbf{r}=(a \mathbf{r}, h)$. The composition of $(a, h)$ and $(b, k)$, defined when $(a \mathbf{r}, h)=(b \mathbf{d},(b \phi) k)$, is then given by $(a, h)(b, k)=(a b, k)$. With due allowance for the change of notation required by Steinberg's use of left actions of groupoids, this is precisely $\operatorname{Der}(\phi)$.

Now $H$ acts on $\operatorname{Der}(\phi)$ : we have $\omega:(a, h) \mapsto h \mathbf{r}$, so that $(a, h) \triangleleft h^{\prime}$ is defined when $h h^{\prime}$ exists, and then $(a, h) \triangleleft h^{\prime}=\left(a, h h^{\prime}\right)$.

Corollary 5.5 ([8], Proposition 4.9). The mapping cocylinder $M^{\phi}$ is isomorphic to the semidirect product $H \ltimes \operatorname{Der}(\phi)$.

Proof. We define $\gamma: H \ltimes \operatorname{Der}(\phi) \rightarrow M^{\phi}$ by $(k,(g, h)) \mapsto\left\langle(g \phi) h k^{-1}, g, h\right\rangle$. Now the composition of $(k,(g, h))$ and $(l,(p, q))$ in $H \ltimes \operatorname{Der}(\phi)$ is defined if and only if $k \mathbf{r}=l \mathbf{d}, g \mathbf{r}=p \mathbf{d}$ and $h=(p \phi) q l^{-1}$, and then we have

$$
(k,(g, h))(l,(p, q))=(k l,(g, h l)(p, q))=(k l,(g p, q)) .
$$

Then

$$
\begin{aligned}
(k,(g, h)) \gamma(l,(p, q)) \gamma & =\left\langle(g \phi) h k^{-1}, g, h\right\rangle\left\langle(p \phi) q l^{-1}, p, q\right\rangle \\
& =\left\langle(g \phi) h k^{-1}, g, h\right\rangle\langle h, p, q\rangle \\
& =\left\langle(g \phi) h k^{-1}, g p, q\right\rangle=\left\langle(g \phi)(p \phi) q l^{-1} k^{-1}, g p, q\right\rangle \\
& =(k l,(g p, q)) \gamma
\end{aligned}
$$


It follows that $\gamma$ is a functor, and $\gamma$ is then easily seen to be an isomorphism of ordered groupoids, with inverse

$$
\gamma^{-1}:\left\langle h_{0}, a, h_{1}\right\rangle \mapsto\left(h_{0}^{-1}(a \phi) h_{1},\left(a, h_{1}\right)\right) .
$$

Remark 5.6. Under the isomorphism of Corollary 5.5, the mapping $p_{\phi}: M^{\phi} \rightarrow H$ corresponds to the projection $\pi: H \ltimes \operatorname{Der}(\phi) \rightarrow H$ and the lifting $\widetilde{F}$ described in the proof of Theorem 5.2 is the same as that given in Lemma4.1.

Take $\phi$ to be the inclusion $H_{0} \hookrightarrow H$. Then $\operatorname{Der}(\phi)$ will be the analogue of the loop space $\Omega H$ of $H$. We then have

$$
\Omega H=\operatorname{Der}(\phi)=\{(h \mathbf{d}, h): h \in H\}
$$

and so $\Omega H$ is identified as the trivial groupoid on the set of arrows of $H$, ordered as a partially ordered set by the ordering on $H$. This is the groupoid $H_{D}$ of [12].

Proposition 5.7. The poset $\Omega H$ is naturally isomorphic to $\operatorname{ker} \varepsilon_{0} \cap \operatorname{ker} \varepsilon_{1}$.

Proof. The kernel of $\varepsilon_{0}$ is the analogue of the set of pointed maps from the unit interval (based at 0 ) to a pointed space $\left(X, x_{0}\right)$. We have

$$
\operatorname{ker} \varepsilon_{0}=\left\{\left[h_{0}, e, h_{1}\right]: e \in H_{0}, h_{0}^{-1} e h_{1} \text { exists }\right\}
$$

and so $\operatorname{OGPD}_{*}(\mathscr{I}, H)$ may be identified with the groupoid whose arrows are pairs of coinitial arrows in $H$, and a pair $\left(h_{0}, h_{1}\right) \in \operatorname{OGPD}_{*}(\mathscr{I}, H)$ then satisfies $\left(h_{0}, h_{1}\right) \mathbf{d}=h_{0},\left(h_{0}, h_{1}\right) \mathbf{r}=h_{1}$ with composition $\left(h_{0}, h_{1}\right)\left(h_{1}, h_{2}\right)=\left(h_{0}, h_{2}\right)$. Then

$$
\varepsilon_{1}: \operatorname{ker} \varepsilon_{0} \rightarrow H,\left(h_{0}, h_{1}\right) \mapsto h_{0}^{-1} h_{1} .
$$

whose kernel is the subset $\{(h, h): h \in H\}$ with $(h, h) \mathbf{d}=h=(h, h) \mathbf{r}$ and $(h, h)(h, h)=(h, h):$ that is $\Omega H$.

Proposition 5.8. The projection $q_{\phi}: M^{\phi} \rightarrow G$ defined by $q:\left\langle h_{0}, a, h_{1}\right\rangle \mapsto a$ is a strong fibration, and its restriction to $\operatorname{Der}(\phi)$ is a covering.

Proof. The map $q_{\phi}$ is a pullback of $\varepsilon_{0}: \operatorname{OGPD}(\mathscr{I}, H) \rightarrow H$ and so is a strong fibration by Propositions 5.1 and 2.3. Using the notational changes introduced in the proof of Corollary 5.4 we have $q_{\phi}: \operatorname{Der}(\phi) \rightarrow G$ given by $(a, h) \mapsto a$, and hence

$$
\operatorname{ker} q_{\phi}=\left\{(e, h): e \in G_{0}, e \phi=h \mathbf{d}\right\}=(\operatorname{Der}(\phi))_{0} .
$$

\section{REFERENCES}

[1] R. Brown, Fibrations of groupoids. J. Algebra 15 (1970) 103-132.

[2] R. Brown, Groupoids as coefficients. Proc. London Math Soc. (3) 25 (1972) 413-426.

[3] R. Brown, P.R. Heath, and K.-H. Kamps, Coverings of groupoids and Mayer-Vietoris type sequences. In Categorical Topology, Proc. Conf. Toledo Ohio 1983 Sigma Ser. Pure Math 5 147162, Heldermann, Berlin (1984). 
[4] C. Ehresmann, Oeuvres Complète et Commentées. (A.C.Ehresmann ed.) Supplements to Cah. Topologie Géom. Différ. Catégoriques (1980-84).

[5] N.D. Gilbert, A P-theorem for ordered groupoids. In Proc. Intl. Conf. Semigroups and Formal Languages, Lisbon 2005 J.M André et al. (Eds.) 84-100. World Scientific (2007).

[6] P.J. Higgins, Notes on categories and groupoids. Van Nostrand Reinhold Math. Stud. 32 (1971). Reprinted electronically at www.tac.mta.co/tac/reprints/articles/7/7tr7.pdf .

[7] M.V. Lawson, Inverse Semigroups. World Scientific (1998).

[8] M.V. Lawson, J. Matthews and T. Porter, The homotopy theory of inverse semigroups. Internat. J. Algebra Comput. 12 (2001) 755-790.

[9] J. Matthews, Topological Ideas in Inverse Semigroup Theory. PhD Thesis, University of Wales, Bangor, (2004).

[10] E.C. Miller, Structure Theorems for Ordered Groupoids. PhD Thesis, Heriot-Watt University, Edinburgh, (2009).

[11] N.R. Reilly, Bisimple $\omega$-semigroups. Proc. Glasgow Math. Assoc. 7 (1966) 160-167.

[12] B. Steinberg, Factorization theorems and morphisms of ordered groupoids and inverse semigroups, Proc. Edin. Math. Soc. 44 (2001) 549-569.

[13] A. Weinstein, Groupoids: unifying internal and external symmetry. Notices Amer. Math. Soc. 43 (1996) 744-752.

[14] G.W. Whitehead, Elements of Homotopy Theory. Grad. Texts in Math. 61, Springer-Verlag (1978).

SCHOol of Mathematical And COMPUTER SCIENCES, AND THE MAXWELl INSTITUTE For the Mathematical Sciences, , Heriot-Watt University, Edinburgh EH14 4AS, U.K

E-mail address: naaa4@hw.ac.uk, N.D.Gilbert@hw.ac.uk, ecm003@hotmail.com 